\title{
PERTURBATIONS OF EMBEDDED EIGENVALUES FOR A MAGNETIC SCHRÖDINGER OPERATOR ON A CYLINDER
}

\author{
ARI LAPTEV AND SARA MAAD SASANE
}

\begin{abstract}
Perturbation problems for operators with embedded eigenvalues are generally challenging since the embedded eigenvalues cannot be separated from the rest of the spectrum. In this paper we study a perturbation problem for embedded eigenvalues for a magnetic Schrödinger operator, when the underlying domain is a cylinder. The magnetic potential is $C^{2}$ with an algebraic decay rate as the unbounded variable of the cylinder tends to $\pm \infty$. In particular, no analyticity of the magnetic potential is assumed. We also assume that the embedded eigenvalue of the unperturbed problem is not the square of an integer, thus avoiding the thresholds of the continuous spectrum of the unperturbed operator. We show that the set of nearby potentials, for which a simple embedded eigenvalue persists, forms a smooth manifold of finite codimension.
\end{abstract}

\section{INTRODUCTION}

Embedded eigenvalues occur in many applications arising in physics. In quantum mechanics, for instance, eigenvalues of the energy operator correspond to energy bound states that can be attained by the underlying physical system (see [24]). When such an operator is perturbed, an embedded eigenvalue typically disappears from the continuous spectrum, while turning into a resonance $[2,5,6,8,9,21,22,23,25]$. On the other hand, in this paper we are interested in studying the structure of the set of perturbations which do not remove an embedded eigenvalue. An abstract setting for a problem of this type is given in [1], and other problems concerning persistence of embedded eigenvalues have been studied in e.g. $[10,13,18,13]$. The current paper builds on methods developed for solving perturbation problems for the bilaplacian [11, 12], but instead of the bilaplacian, we study in this paper a perturbation problem for a magnetic Schrödinger operator on a cylindrical domain, where the small perturbation enters as an addition to the magnetic potential. Other perturbation problems for embedded eigenvalues of magnetic Schrödinger operators have been studied in e.g. [5, 7].

More specifically, we consider the elliptic operator

$$
\mathcal{L}_{A_{0}}:=-\partial_{z}^{2}-\left(\partial_{\varphi}+i A_{0}(z, \varphi)\right)^{2}
$$

acting on $L^{2}\left(\mathbb{R} \times S^{1}\right)$ where $A_{0}: \mathbb{R} \times S^{1} \rightarrow \mathbb{R}$ decays to 0 as $|z| \rightarrow \infty$. $\mathcal{L}_{A_{0}}$ is self-adjoint, and for the $A_{0}$ we are considering, the essential spectrum of $\mathcal{L}_{A_{0}}$ is $[0, \infty)$. Furthermore, we choose $A_{0}$ so that $\mathcal{L}_{A_{0}}$ has an eigenvalue $\lambda_{0}>0$. In this situation, we say that $\lambda_{0}$ is an embedded eigenvalue of $\mathcal{L}_{A_{0}}$, since $\lambda_{0}$ is an eigenvalue which also belongs to the continuous spectrum of $\mathcal{L}_{A_{0}}$. The domain of $\mathcal{L}_{A_{0}}$ is $H_{A_{0}}^{2}=\left\{u \in L^{2}\left(\mathbb{R} \times S^{1}\right) ; \mathcal{L}_{A_{0}} u \in L^{2}\left(\mathbb{R} \times S^{1}\right)\right\}$. We first show that there exists such a potential $A_{0}$.

Example 1. It is possible to choose $A_{0}(z, \varphi)=A_{0}(z)$, i.e. $A_{0}$ is independent of $\varphi$, in such a way that $\mathcal{L}_{A_{0}}$ has an embedded eigenvalue $\lambda_{0}$ with corresponding eigenfunction of the form $u(z, \varphi)=v(z) e^{i n \varphi}$.

Indeed, $u_{*}(z, \varphi)=v(z) e^{i n \varphi}$ satisfies the eigenvalue equation if and only if $v$ satisfies the onedimensional equation

$$
-v^{\prime \prime}(z)+\left(n+A_{0}(z)\right)^{2} v(z)=\lambda_{0} v(z) .
$$

It suffices to find functions $v \in H^{2}(\mathbb{R})$ and $A_{0}(z)$, such that $A_{0}(z) \rightarrow 0$ as $|z| \rightarrow \infty$ and which satisfy the above equation. It is clear that there are many such pairs of functions. For a concrete

Date: December 19, 2016. 
example, we take

$$
\begin{aligned}
\lambda_{0} & =n^{2}-1 \\
A_{0}(z) & =-n+\sqrt{n^{2}-\frac{2}{\cosh ^{2}(z)}} \\
v(z) & =\frac{1}{\cosh (z)} .
\end{aligned}
$$

It is straight-forward to verify that this choice of $\lambda_{0}$ and $A_{0}, v$ solves (1). If we choose $n \geq 2$, then $A_{0}(z)$ is real and $\lambda_{0}=n^{2}-1>0$ is an embedded eigenvalue.

In this paper, we assume that $\mathcal{L}_{A_{0}}$ has an embedded eigenvalue $\lambda_{0}$ of multiplicity 1 with a corresponding eigenfunction $u_{*}$. $A_{0}$ may depend on $\varphi$ and $A_{0}(z, \varphi) \rightarrow 0$ as $|z| \rightarrow \infty$, with a decay rate that will be specified below. Next, we replace $A_{0}$ by $A(z, \varphi)$, where $A$ is close to $A_{0}$ in a function space defined below, and ask the question whether the new operator $\mathcal{L}_{A}:=$ $-\partial_{z}^{2}-\left(\partial_{\varphi}+i A(z, \varphi)\right)^{2}$ has an embedded eigenvalue $\lambda$ close to $\lambda_{0}$. We note that the essential spectrum of $\mathcal{L}_{A}$ is the same as that of $\mathcal{L}_{A_{0}}$.

\section{MAin ReSUlt}

We assume:

(A1) $A_{0} \in C^{2}\left(\mathbb{R} \times S^{1}\right)$ and

$$
\sup _{(z, \varphi) \in \mathbb{R} \times S^{1}}\left(\left|\frac{\partial^{2} A_{0}}{\partial z^{2}}(z, \varphi)\right|+\left|\frac{\partial^{2} A_{0}}{\partial z \partial \varphi}(z, \varphi)\right|+\left|\frac{\partial^{2} A_{0}}{\partial \varphi^{2}}(z, \varphi)\right|\right)<\infty .
$$

(A2) There exists a $\beta>1$ such that

$$
\sup _{(z, \varphi) \in \mathbb{R} \times S^{1}}\left(\left|A_{0}(z, \varphi)\right|+\left|\frac{\partial A_{0}}{\partial \varphi}(z, \varphi)\right|\right)(1+|z|)^{\beta}<\infty .
$$

(A3) There exists a $z_{0} \in \mathbb{R}$ such that $\int_{0}^{2 \pi} A_{0}\left(z_{0}, \varphi\right) d \varphi \neq 0$.

Remark 1. These assumptions are all satisfied for the equation of Example 1. Indeed, (A1) and (A2) is satisfied, which can be verified by differentiation, and (A3) is trivially satisfied, since $A_{0} \not \equiv 0$ is independent of $\varphi$.

We define the Banach space $X_{\beta}$ to consist of the functions $A \in C^{2}\left(\mathbb{R} \times S^{1}\right)$ for which

$$
\begin{aligned}
\|A\|_{X_{\beta}}: & =\sup _{(z, \varphi) \in \mathbb{R} \times S^{1}}\left(\left|\frac{\partial^{2} A}{\partial z^{2}}(z, \varphi)\right|+\left|\frac{\partial^{2} A}{\partial z \partial \varphi}(z, \varphi)\right|+\left|\frac{\partial^{2} A}{\partial \varphi^{2}}(z, \varphi)\right|\right) \\
& +\sup _{(z, \varphi) \in \mathbb{R} \times S^{1}}\left(|A(z, \varphi)|+\left|\frac{\partial A}{\partial \varphi}(z, \varphi)\right|\right)(1+|z|)^{\beta}<\infty .
\end{aligned}
$$

Our goal is to analyze persistence of the eigenvalue $\lambda_{0}$ when $A_{0}$ is replaced by the nearby potential $A$ (in $X_{\beta}$ ). Thus, we consider the operator $\mathcal{L}_{A}$ and wish to characterize the set

$$
\mathcal{M}_{\delta}=\left\{A \in X_{\beta} ; \text { there exists } \lambda \in\left(\lambda_{0}-\delta, \lambda_{0}+\delta\right) \text { such that } \lambda \text { is an eigenvalue of } \mathcal{L}_{A}\right\}
$$

in a neighbourhood of $A=A_{0}$ for small $\delta>0$. Our main result is:

Theorem 1. Let $\lambda_{0}>0$ be an eigenvalue of $\mathcal{L}_{A_{0}}$ of multiplicity 1. Suppose that (A1) and (A2) hold, and let $m$ be a positive integer such that $(m-1)^{2}<\lambda_{0}<m^{2}$. Then there exists a $\delta>0$ and a neighbourhood $\mathcal{O}$ of $A_{0} \in X_{\beta}$ such that $\mathcal{M}_{\delta} \cap \mathcal{O}$ is a smooth manifold of codimension $4 m-2$.

Next, we comment on the assumption that $\lambda_{0}$ has to avoid the square of the integers. Note that the squares of the integers have to be excluded for $\lambda_{0}$, since from the conclusion of the theorem, the co-dimension of the manifold would also change at these points, at least if the eigenvalue $\lambda$ for the operator $\mathcal{L}_{A}$ would be on different sides of $\lambda_{0}$ for different $A$ close to $A_{0}$. The number $4 m-2$ 
is the multiplicity of the continuous spectrum of $\mathcal{L}_{A}$ at $\lambda \in\left((m-1)^{2}, m^{2}\right)$. Intuitively, we can think of the continuous spectrum as being generated by the continuum eigenfunctions

$$
u(z, \varphi)=e^{i k z} e^{i n \varphi}, \quad k \in \mathbb{R}, n \in \mathbb{Z}
$$

of $-\Delta$, which satisfy $-\Delta u=\left(k^{2}+n^{2}\right) u$ and can be turned into approximate eigenfunctions of $\mathcal{L}_{A}$ by shifting them along the $z$-direction towards $\infty$, and truncating them using cutoff functions. Note that if $(m-1)^{2}<\lambda<m^{2}$ for some $m \in \mathbb{Z}$, then $-\Delta$ admits $4 m-2$ linearly independent continuum eigenfunctions which are obtained by choosing $|n|=0, \ldots, m-1$ in (2) and afterwards $k \in \mathbb{R}$ so that $\lambda=k^{2}+n^{2}$. Thus, it is reasonable to refer to $4 m-2$ as the multiplicity of the continuous spectrum at $\lambda \in\left((m-1)^{2}, m^{2}\right)$. A rigorous definition of the multiplicity of the continuous spectrum can be found in [3], pp. 59-60, and using the spectral resolution of the Fourier transform of $-\Delta$ on $L^{2}$, it is not difficult to see that this definition also gives $4 m-2$, thus justifying the preceding formal calculation.

\section{The ODE FORMULATiOn}

We view the function $u$ as a function of one variable $z$, taking values in a space of functions on the circle $S^{1}$. Let $\partial: H^{j}\left(S^{1}\right) \rightarrow H^{j-1}\left(S^{1}\right)$ denote differentiation with respect to $\varphi$, where $H^{j}\left(S^{1}\right)$ is the Sobolev space of complex valued functions whose weak derivatives up to order $j$ are square integrable. By ' we mean differentiation with respect to $z$. Consider $\lambda \in\left((m-1)^{2}, m^{2}\right)$, where $m$ is a positive integer. After putting $u=u_{1}, u^{\prime}=u_{2}$, the eigenvalue equation can be rewritten as the system

$$
U^{\prime}=B(z ; \lambda, A) U
$$

where $U=\left(u_{1}, u_{2}\right)^{T}$, and

$$
B(z ; \lambda, A)=\left[\begin{array}{cc}
0 & 1 \\
-(\partial+i A(z))^{2}-\lambda & 0
\end{array}\right] .
$$

The magnetic potential $A$ acts on functions in $C\left(\mathbb{R} ; H^{k}\left(S^{1}\right)\right)$ as follows: $A u(z):=A(z, \cdot) u(z)$. The matrix $B(z ; \lambda, A)$ is an unbounded operator on $X:=H^{1}\left(S^{1}\right) \times L^{2}\left(S^{1}\right)$ with domain $Y:=$ $H^{2}\left(S^{1}\right) \times H^{1}\left(S^{1}\right)$. The norm on $X$ is defined by

$$
\|U\|_{X}^{2}=\left\|u_{1}\right\|_{H^{1}}^{2}+\left\|u_{2}\right\|_{L^{2}}^{2} .
$$

We can now prove the following lemma:

Lemma 1. $u \in H_{\text {loc }}^{2}\left(\mathbb{R} \times S^{1}\right)$ satisfies

$$
\left(-\partial_{z}^{2}-\left(\partial_{\varphi}+i A(z, \varphi)\right)^{2}\right) u=\lambda u
$$

if and only if the ODE system (3) has a solution $U \in C(\mathbb{R} ; Y) \cap C^{1}(\mathbb{R} ; X)$.

Proof. Suppose that $u \in H_{\text {loc }}^{2}\left(\mathbb{R} \times S^{1}\right)$ satisfies the equation $\mathcal{L}_{A} u=\lambda u$. Since $A \in X_{\beta}$, we have in particular that $A \in C^{2}\left(\mathbb{R} \times S^{1} ; \mathbb{R}\right)$, and it follows that

$$
-\partial_{z}^{2} u-\partial_{\varphi}^{2} u=\lambda u+i A_{\varphi}^{\prime}(z, \varphi) u+2 i A(z, \varphi) \partial_{\varphi} u+A(z, \varphi)^{2} u \in H_{l o c}^{1}\left(\mathbb{R} \times S^{1}\right),
$$

and so $u \in H_{l o c}^{3}\left(\mathbb{R} \times S^{1}\right)$ by elliptic regularity theory. When viewing $u$ as a function of $z$ with values in a Sobolev space on the circle, it is clear that

$$
u \in H_{l o c}^{1}\left(\mathbb{R} ; H^{2}\left(S^{1}\right)\right) \cap H_{l o c}^{2}\left(\mathbb{R} ; H^{1}\left(S^{1}\right)\right) \cap H_{l o c}^{3}\left(\mathbb{R} ; L^{2}\left(S^{1}\right)\right) .
$$

By the Sobolev embedding theorem, we have that $u_{1}=u \in C\left(\mathbb{R} ; H^{2}\left(S^{1}\right)\right) \cap C^{1}\left(\mathbb{R} ; H^{1}\left(S^{1}\right)\right)$, while $u_{2}=u^{\prime} \in C\left(\mathbb{R} ; H^{1}\left(S^{1}\right)\right) \cap C^{1}\left(\mathbb{R} ; L^{2}\left(S^{1}\right)\right)$. It follows that $U \in C(\mathbb{R} ; Y) \cap C^{1}(\mathbb{R} ; X)$. It is also clear that $U$ satisfies the system (3).

Conversely, if $U \in C(\mathbb{R} ; Y) \cap C^{1}(\mathbb{R} ; X)$ is a solution of (3), we let $u=U_{1}$ and note that $u \in C\left(\mathbb{R} ; H^{2}\left(S^{1}\right)\right) \cap C^{1}\left(\mathbb{R} ; H^{1}\left(S^{1}\right)\right) \subset H_{\text {loc }}^{1}\left(\mathbb{R} \times S^{1}\right)$. It is also clear that $u$ is a weak solution of $\mathcal{L}_{A} u=\lambda u$. By elliptic regularity theory (e.g. [14], Theorem 8.8, p. 183) it follows that $u \in H_{l o c}^{2}\left(\mathbb{R} \times S^{1}\right)$, and so the proof is complete. 
3.1. The system at infinity. We will see in Lemma 6 that the eigenfunctions of $\mathcal{L}_{A}$ are exponentially decaying as $|z| \rightarrow \infty$. The corresponding solutions of (3) turn out to be asymptotic to exponentially decaying solutions of the system at infinity defined below. By assumption (A2), $\max _{\varphi \in S^{1}}\left(|A(z, \varphi)|+\left|A_{\varphi}^{\prime}(z, \varphi)\right|\right) \rightarrow 0$ as $|z| \rightarrow \infty$. It is then natural to define the system at infinity to be the equation

$$
U^{\prime}=B_{\infty}(\lambda) U
$$

where

$$
B_{\infty}(\lambda)=\left[\begin{array}{cc}
0 & 1 \\
-\partial^{2}-\lambda & 0
\end{array}\right]
$$

To analyze the solutions of (5), the function $U=\left(u_{1}, u_{2}\right)^{T}$ is expanded as a Fourier series in the $\varphi$ variable, i.e. $U(z)(\varphi)=\sum_{k \in \mathbb{Z}} \widehat{U}_{k}(z) e^{i k \varphi}$. Then for $k \in \mathbb{Z}$,

$$
\widehat{U}_{k}^{\prime}(z)=\widehat{B}_{\infty}(k ; \lambda) \widehat{U}_{k}(z),
$$

where

$$
\widehat{B}_{\infty}(k ; \lambda)=\left(\begin{array}{cc}
0 & 1 \\
k^{2}-\lambda & 0
\end{array}\right) .
$$

For any $k \in \mathbb{Z}$, the eigenvalues of $B_{\infty}(k ; \lambda)$ are $\pm \sqrt{k^{2}-\lambda}$, and the corresponding eigenvectors are $\left(1, \pm \sqrt{k^{2}-\lambda}\right)^{T}$. Here, and in the rest of the paper, we are using the principal square root so that e.g. $\sqrt{-1}=i$.

We define

$$
M_{k}(\lambda):=\left(\begin{array}{cc}
-1 / \sqrt{k^{2}-\lambda} & 1 / \sqrt{k^{2}-\lambda} \\
1 & 1
\end{array}\right)
$$

and

$$
D_{k}:=\left(\begin{array}{cc}
-\sqrt{k^{2}-\lambda} & 0 \\
0 & \sqrt{k^{2}-\lambda}
\end{array}\right)
$$

so that $\widehat{B}_{\infty}(k ; \lambda)=M_{k}(\lambda) D_{k}(\lambda) M_{k}^{-1}(\lambda)$.

We can now show the following:

Lemma 2. Let $\lambda \in\left((m-1)^{2}, m^{2}\right)$, where $m$ is a positive integer. Then $B_{\infty}(\lambda): X \rightarrow X$ is closed and densely defined, and

$$
\sigma\left(B_{\infty}(\lambda)\right)=\left\{ \pm \sqrt{k^{2}-\lambda} ; k \in \mathbb{Z}\right\} .
$$

In other words, $B_{\infty}(\lambda)$ has $m$ pairs of purely imaginary eigenvalues $\mu= \pm i \sqrt{\lambda-k^{2}}, k=0, \ldots, m-$ 1. All other eigenvalues are real, and they are given by $\pm \sqrt{k^{2}-\lambda}, k \geq m$. All eigenvalues with $k \neq 0$ have a two-dimensional eigenspace. The smallest positive eigenvalue of $B_{\infty}(\lambda)$ is $\mu_{\min }(\lambda):=\sqrt{m^{2}-\lambda}$.

Proof. It is clear that $Y:=H^{2}\left(S^{1}\right) \times H^{1}\left(S^{1}\right)=\operatorname{Dom}\left(B_{\infty}(\lambda)\right)$ is dense in $X$. In order to prove that $B_{\infty}(\lambda)$ is a closed operator, we let $U_{j} \in Y$, and $U, f \in X$ be such that $U_{j} \stackrel{X}{\rightarrow} U$ and $B_{\infty}(\lambda) U_{j} \stackrel{X}{\rightarrow} f$. By the definition of $B_{\infty}(\lambda)$, we see that $f_{1}=u_{2}$ and $u_{2, j} \stackrel{H^{1}}{\rightarrow} u_{2}$. Moreover, $u_{1, j} \stackrel{H^{1}}{\rightarrow} u_{1}$ and $-\partial^{2} u_{1, j} \stackrel{L^{2}}{\rightarrow} f_{2}+\lambda u_{1}$. It follows that $u_{1, j} \stackrel{H^{2}}{\rightarrow} u_{1}$ and $-\partial^{2} u_{1}-\lambda u_{1}=f_{2} \in L^{2}$. Hence $U \in Y$ and $B_{\infty}(\lambda) U=f$. This shows that $B_{\infty}(\lambda)$ is closed in $X$.

Let $l_{s}^{2}:=\left\{f: \mathbb{Z} \rightarrow \mathbb{R} ; \sum_{k=-\infty}^{\infty}\left(1+k^{2}\right)^{s}|f(k)|^{2}<\infty\right\}$, and let $\widehat{X}:=l_{1}^{2} \times l^{2}$ and $\widehat{Y}:=l_{2}^{2} \times l_{1}^{2}$. Note that $U \in X$ if and only if the coefficients of its Fourier series $\widehat{U} \in \widehat{X}$, etc.

Define the operator $\widehat{B}_{\infty}(\lambda): \widehat{X} \rightarrow \widehat{X}$ by

$$
\left(\widehat{B}_{\infty}(\lambda) \widehat{U}\right)_{k}:=\widehat{B}_{\infty}(k ; \lambda) \widehat{U}_{k},
$$

and note that $\widehat{B}_{\infty}(\lambda)$ is closed and densely defined with domain $\widehat{Y}$. It is clear that $\left(B_{\infty}(\lambda)-\mu I\right)$ : $X \rightarrow X$ has a bounded inverse if and only if $\left(\widehat{B}_{\infty}(\lambda)-\mu I\right): \widehat{X} \rightarrow \widehat{X}$ has a bounded inverse. It is also clear that the eigenvalues of $\widehat{B}_{\infty}(k ; \lambda)$ belong to the spectrum of $\widehat{B}_{\infty}(\lambda)$. For $k \geq 1$, 
the operators $\widehat{B}_{\infty}(k ; \lambda)$ and $\widehat{B}_{\infty}(-k ; \lambda)$ have the same eigenvalues, and so the corresponding eigenspaces of $\widehat{B}_{\infty}(\lambda)$ are 2-dimensional.

We need to show that there are no other points in the spectrum. Define $\widehat{M}: l^{2} \times l^{2} \rightarrow \widehat{X}$ by

$$
(\widehat{M} \widehat{U})_{k}=M_{k} \widehat{U}_{k}
$$

and note that $\widehat{M}$ is a linear homeomorphism between these spaces. Define also the unbounded operator $\widehat{D}$ on $l^{2} \times l^{2}$ by

$$
(\widehat{D} \widehat{U})_{k}=D_{k} \widehat{U}_{k}
$$

Note that $\widehat{D}$ is closed and densely defined with domain $l_{1}^{2} \times l_{1}^{2}$, and that

$$
\sigma(\widehat{D})=\cup_{k \in \mathbb{Z}} \sigma\left(\widehat{B}_{\infty}(k, \lambda)\right) .
$$

If $\mu \notin \sigma(\widehat{D})$, then

$$
\left(\widehat{B}_{\infty}(\lambda)-\mu I\right)^{-1}=\widehat{M}(\widehat{D}-\mu I)^{-1} \widehat{M}^{-1},
$$

and so $\left(\widehat{B}_{\infty}(\lambda)-\mu\right)^{-1}$ is bounded. The proof is complete.

Let $X^{u}(\lambda)$ and $X^{s}(\lambda)$ be the closure in $X$ of the span of the eigenvectors of $B_{\infty}(\lambda)$ corresponding to the positive and the negative eigenvalues of $B_{\infty}(\lambda)$, respectively. We denote by $X^{c}(\lambda)$ the span of the eigenvalues corresponding to the purely imaginary eigenvalues. By Lemma $2, X=$ $X^{s}(\lambda) \oplus X^{c}(\lambda) \oplus X^{u}(\lambda)$. Note that $X^{u}(\lambda)$ and $X^{s}(\lambda)$ are infinite dimensional while $X^{c}(\lambda)$ is finite dimensional (with dimension $4 m-2$ ). Let $P^{u}(\lambda), P^{c}(\lambda)$ and $P^{s}(\lambda)$ be the spectral projections onto $X^{u}(\lambda), X^{s}(\lambda)$ and $X^{c}(\lambda)$, respectively.

The operators $B_{\infty}(\lambda)$ and $-B_{\infty}(\lambda)$ do not generate semigroups, since the spectrum of $B_{\infty}(\lambda)$ is unbounded both on the right and the left half planes. The following lemma shows that after projecting onto $X^{s}(\lambda), X^{c}(\lambda)$ and $X^{u}(\lambda)$, we obtain analytic semigroups on these respective spaces:

Lemma 3. Let $\lambda \in\left((m-1)^{2}, m^{2}\right)$. Then the operators $B_{\infty}(\lambda) P^{s}(\lambda),-B_{\infty}(\lambda) P^{u}(\lambda), B_{\infty}(\lambda)(I-$ $\left.P^{u}(\lambda)\right)$ and $-B_{\infty}(\lambda)\left(I-P^{s}(\lambda)\right)$ generate analytic semigroups on $X^{s}(\lambda), X^{u}(\lambda), X^{s}(\lambda) \oplus X^{c}(\lambda)$, and $X^{u}(\lambda) \oplus X^{c}(\lambda)$, respectively.

Proof. Let $\eta \in\left(0, \mu_{\min }(\lambda)\right)$, where we recall that $\mu_{\min }(\lambda)=\sqrt{m^{2}-\lambda}$ is the smallest positive eigenvalue of $B_{\infty}(\lambda)$. We define $V_{ \pm}(z):=e^{ \pm \eta z} U(z)$, where $U$ solves (3), and note that $V_{ \pm}$satisfies the system

$$
V_{ \pm}^{\prime}=\left(B_{\infty}(\lambda) \pm \eta I\right) V_{ \pm} .
$$

It suffices to show that there exists a constant $C>0$ such that

$$
\left\|\left(B_{\infty}(\lambda) \pm \eta I-i \mu I\right)^{-1}\right\| \leq \frac{C}{1+|\mu|}
$$

for every $\mu \in \mathbb{R}$. As in the proof of Lemma 2 , we note that this estimate follows if there exists a constant $\widetilde{C}>0$ such that for every $\widehat{U} \in l^{2} \times l^{2}$

$$
\left\|(\widehat{D} \pm \eta I-i \mu I)^{-1} \widehat{U}\right\|_{l^{2} \times l^{2}} \leq \frac{\widetilde{C}}{(1+|\mu|)}\|\widehat{U}\|_{l^{2} \times l^{2}}
$$

(cf. [19], p. 61). For $k \in \mathbb{Z}$ we have

$$
\left(D_{k} \pm \eta I-i \mu I\right)^{-1} \widehat{U}_{k}=\left(\frac{\widehat{u}_{k, 1}}{-\sqrt{k^{2}-\lambda} \pm \eta-i \mu}, \frac{\widehat{u}_{k, 2}}{\sqrt{k^{2}-\lambda} \pm \eta-i \mu}\right)^{T},
$$

and so if $k^{2} \geq m^{2}$,

$$
\begin{aligned}
\left|\left(D_{k} \pm \eta I-i \mu I\right)^{-1} \widehat{U}_{k}\right|^{2} & =\frac{\left|\widehat{u}_{k, 1}\right|^{2}}{\left(-\sqrt{k^{2}-\lambda} \pm \eta\right)^{2}+\mu^{2}}+\frac{\left|\widehat{u}_{k, 2}\right|^{2}}{\left(\sqrt{k^{2}-\lambda} \pm \eta\right)^{2}+\mu^{2}} \\
& \leq \frac{\left|\widehat{U}_{k}\right|^{2}}{\left(\sqrt{m^{2}-\lambda}-\eta\right)^{2}+\mu^{2}} \leq \frac{2\left|\widehat{U}_{k}\right|^{2}}{\left(\sqrt{m^{2}-\lambda}-\eta+|\mu|\right)^{2}} \leq \frac{\widetilde{C}^{2}}{(1+|\mu|)^{2}}\left|\widehat{U}_{k}\right|^{2},
\end{aligned}
$$


whereas if $k^{2} \leq(m-1)^{2}$, clearly

$$
\left|\left(D_{k} \pm \eta I-i \mu I\right)^{-1} \widehat{U}_{k}\right|^{2} \leq \frac{C_{k}^{2} \widehat{U}_{k}^{2}}{(1+|\mu|)^{2}}
$$

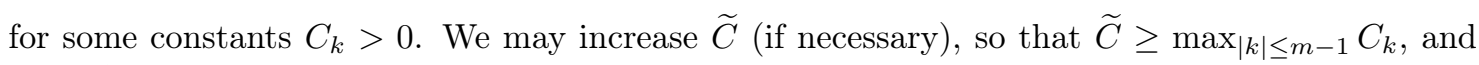
so we have

$$
\left|\left(D_{k} \pm \eta I-i \mu I\right)^{-1} \widehat{U}_{k}\right|^{2} \leq \frac{\widetilde{C}^{2}}{(1+|\mu|)^{2}}\left|\widehat{U}_{k}\right|^{2}
$$

for all $k \in \mathbb{Z}$. Summing over $k \in \mathbb{Z}$ yields the desired inequality.

3.2. Exponential dichotomies. We define exponential dichotomies following [20].

Definition 1. Let $J=\mathbb{R}_{-}, \mathbb{R}_{+}$or $\mathbb{R}$. An ODE system $U^{\prime}=B(z) U$, where $U(z) \in X$ and $B(z): X \rightarrow X$ is said to possess an exponential dichotomy on $J$ if there exists a family of projections $P(z) \in \mathcal{L}(X), z \in J$, such that the projections satisfy $P(\cdot) U \in C(J ; X)$ for any $U \in X$, and there exist constants $K$ and $\kappa^{s}<0<\kappa^{u}$ with the following properties:

(i) For any $z_{0} \in J$ and $U \in X$ there exists a unique solution $\Phi^{s}\left(z, z_{0}\right) U$ of (3) defined for $z \geq z_{0}, z, \zeta \in J$, such that $\Phi^{s}\left(z_{0}, z_{0}\right) U=P\left(z_{0}\right) U$ and

$$
\left\|\Phi^{s}\left(z, z_{0}\right) U\right\|_{X} \leq K e^{\kappa^{s}\left(z-z_{0}\right)}\|U\|_{X}
$$

for every $z \geq z_{0}, z, z_{0} \in J$.

(ii) For any $z_{0} \in J$ and $U \in X$ there exists a unique solution $\Phi^{u}\left(z, z_{0}\right) U$ defined for $z \leq z_{0}$, $z, z_{0} \in J$ such that $\Phi^{u}\left(z_{0}, z_{0}\right) U=\left(I-P\left(z_{0}\right)\right) U$ and

$$
\left\|\Phi^{u}\left(z, z_{0}\right) U\right\|_{X} \leq K e^{\kappa^{u}\left(z-z_{0}\right)}\|U\|_{X}
$$

for every $z \leq z_{0}, z, z_{0} \in J$.

(iii) The solutions $\Phi^{s}\left(z, z_{0}\right) U$ and $\Phi^{u}\left(z, z_{0}\right) U$ satisfy

$$
\begin{aligned}
& \Phi^{s}\left(z, z_{0}\right) U \in \operatorname{Ran} P(z) \quad \text { for all } z \geq z_{0}, z, z_{0} \in J, \\
& \Phi^{u}\left(z, z_{0}\right) U \in \operatorname{ker} P(z) \quad \text { for all } z \leq z_{0}, z, z_{0} \in J .
\end{aligned}
$$

With the above definition, the following corollary is immediate from Lemma 3 :

Corollary 1. Let $\lambda \in\left((m-1)^{2}, m^{2}\right)$ and suppose that $\eta \in\left(0, \mu_{\min }(\lambda)\right)$. Then the operator $B_{\infty}(\lambda)+\eta I$ possesses an exponential dichotomy on $\mathbb{R}$ with $\kappa^{s}=\mu_{\min }(\lambda)+\eta$ and $\kappa^{u}=\eta$, while the operator $B_{\infty}(\lambda)-\eta I$ possesses an exponential dichotomy on $\mathbb{R}$ with $\kappa^{s}=-\eta$ and $\kappa^{u}=\mu_{\min }(\lambda)-\eta$.

Proof. For $B_{\infty}(\lambda)+\eta I$, put $P(z)=P^{s}(\lambda)$ and

$$
\begin{aligned}
\Phi^{s}\left(z, z_{0}\right) & =e^{P^{s}(\lambda)\left(B_{\infty}(\lambda)+\eta I\right)\left(z-z_{0}\right)} P^{s}(\lambda), \\
\Phi^{u}\left(z, z_{0}\right) & =e^{\left(I-P^{s}(\lambda)\right)\left(B_{\infty}(\lambda)+\eta I\right)\left(z-z_{0}\right)}\left(I-P^{s}(\lambda)\right),
\end{aligned}
$$

and for $B_{\infty}(\lambda)-\eta I$, put $P(z)=I-P^{u}(\lambda)$ and

$$
\begin{aligned}
& \Phi^{s}(z, \zeta)=e^{\left(I-P^{u}(\lambda)\right)\left(B_{\infty}(\lambda)-\eta I\right)\left(z-z_{0}\right)}\left(I-P^{u}(\lambda)\right), \\
& \Phi^{u}(z, \zeta)=e^{P^{u}(\lambda)\left(B_{\infty}(\lambda)-\eta I\right)\left(z-z_{0}\right)} P^{u}(\lambda) .
\end{aligned}
$$

It is clear that these two operators satisfy the conditions of exponential dichotomies.

Next, we study the full system (3), which can be expressed as

$$
U^{\prime}=\left(B_{\infty}(\lambda)+L(z ; A)\right) U
$$

where

$$
L(z ; A)=\left[\begin{array}{cc}
0 & 0 \\
A(z, \cdot)^{2}-i A_{\varphi}^{\prime}(z, \cdot)-2 i A(z, \cdot) \partial & 0
\end{array}\right] .
$$

To be able to define exponential dichotomies, we consider an exponentially weighted variable $V_{ \pm}=e^{ \pm \eta z} U(z)$. Then $V_{ \pm}$satisfies the equation

$$
V_{ \pm}^{\prime}=\left(B_{\infty}(\lambda) \pm \eta I+L(z ; A)\right) V_{ \pm} .
$$

These systems have exponential dichotomies on $\mathbb{R}_{ \pm}$. 
Lemma 4. Let $\lambda \in\left((m-1)^{2}, m^{2}\right)$, and suppose that (A1) and (A2) are satisfied. Then for any $\eta \in\left(0, \mu_{\min }(\lambda)\right)$, the systems (9) have exponential dichotomies on $\mathbb{R}_{+}$and $\mathbb{R}_{-}$:

(i) The system for $V_{+}$has an exponential dichotomy with rates $\kappa^{s}=-\mu_{\min }(\lambda)+\eta$ and $\kappa^{u}=\eta$.

(ii) The system for $V_{-}$has an exponential dichotomy with rates $\kappa^{s}=\eta$ and $\kappa^{u}=\mu_{\min }(\lambda)-\eta$.

For $V_{+}$, the projection on $\mathbb{R}_{+}$is denoted by $P_{+}^{s}(\cdot ; \lambda, A)$ and the projection on $\mathbb{R}_{-}$is denoted by $I-P_{-}^{c u}(\cdot ; \lambda, A)$. For $V_{-}$, the projection on $\mathbb{R}_{-}$is denoted by $I-P_{-}^{u}(\cdot ; \lambda, A)$ and the projection on $\mathbb{R}_{+}$is denoted by $P_{+}^{c s}(\cdot ; \lambda, A)$. The corresponding evolution operators are denoted by $\Psi_{+}^{s}(\cdot, \cdot ; \lambda, A)$ and $\Psi_{+}^{c u}(\cdot, \cdot ; \lambda, A)$ for $V_{+}$on $\mathbb{R}_{+}$, etc. Moreover, the evolution operators depend smoothly on $\lambda$ and A.

Proof. The result follows from [20, Theorem 1] if we can verify conditions (H1), (H2), (H3) and (H5) of the same paper. In Lemma 3 we have seen that (H1) holds, namely that there exists a constant $C>0$ such that

$$
\left\|\left(B_{\infty}(\lambda) \pm \eta I-i \mu\right)^{-1}\right\|_{\mathcal{L}(X)} \leq \frac{C}{1+|\mu|} .
$$

Next, we verify (H2) with $\alpha=0$ of the same paper, namely that there exist $\theta>0, t_{*} \geq 0$, and $S, K \in C^{0, \theta}(J, L(X))$ with $L(z ; A)=S(z)+K(z)$ such that $\|S(z)\|_{\mathcal{L}(X, X)} \leq \epsilon$ for $z \in J$, and $K(z)=0$ for all $z \in J$ with $|z| \geq t_{*} . A$ is clearly Hölder continuous in the $z$ variable and since $\|L(z, A)\|_{\mathcal{L}(X)} \rightarrow 0$ as $|z| \rightarrow \infty$, it is easy to see that (H2) holds. We also remark that $\epsilon$ can be taken arbitrarily small.

The condition (H3) is that $\left(B_{\infty}(\lambda) \pm \eta I\right)^{-1}$ is compact in $\mathcal{L}(X)$. This follows since $\operatorname{Dom}\left(B_{\infty}(\lambda)\right)=$ $Y$ is compactly embedded in $X$.

It remains to check condition (H5), namely that $U(t)=0(W(t)=0)$ is the only bounded solution of (3) (or its adjoint equation $W^{\prime}=-B(z ; \lambda, A)^{*} W$ ), satisfying the initial condition $U(0)=0(W(0)=0)$. This follows from Theorem 2.5 of [17].

Smoothness of the exponential dichotomies with respect to the parameters $(\lambda, A)$ can be shown by the implicit function theorem in a neighbourhood of a point $(\lambda, A)$.

Using Lemma 4, we define the following evolution operators for the system (8) on $\mathbb{R}_{+}$and $\mathbb{R}_{-}$:

$$
\begin{array}{cl}
\Phi_{ \pm}\left(z, z_{0} ; \lambda, A\right)=e^{-\eta\left(z-z_{0}\right)} \Psi_{ \pm}^{s}\left(z, z_{0} ; \lambda, A\right), & \Phi_{ \pm}^{c u}\left(z, z_{0} ; \lambda, A\right)=e^{-\eta\left(z-z_{0}\right)} \Psi_{ \pm}^{c u}\left(z, z_{0} ; \lambda, A\right), \\
\Phi_{ \pm}^{u}\left(z, z_{0} ; \lambda, A\right)=e^{\eta\left(z-z_{0}\right)} \Psi_{ \pm}^{u}\left(z, z_{0} ; \lambda, A\right), & \Phi_{ \pm}^{c s}\left(z, z_{0} ; \lambda, A\right)=e^{\eta\left(z-z_{0}\right)} \Psi_{ \pm}^{c s}\left(z, z_{0} ; \lambda, A\right) .
\end{array}
$$

\section{Exponential DECAY OF EIGENFUnCTIONS}

In this section we show that every eigenfunction is exponentially decaying. Our first lemma gives an expression for the bounded solutions of (3). This expression is then used to prove that the eigenfunctions decay exponentially. To avoid cumbersome notation, we write $P^{s}$ instead of $P^{s}(\lambda)$, etc, in the formulas below.

Lemma 5. Let $U$ be a solution of (3). Then

(i) if $U$ is bounded on $\mathbb{R}_{+}$, then for every $R \geq 0$ there exist a $U_{0}^{s} \in X^{s}$ and a $U_{0}^{c} \in X^{c}$ such that for $z \geq R$

$$
\begin{gathered}
U(z)=e^{B_{\infty} P^{s}(z-R)} U_{0}^{s}+e^{B_{\infty} P^{c} z} U_{0}^{c}+\int_{R}^{z} e^{B_{\infty} P^{s}(z-\xi)} P^{s} L(\xi ; A) U(\xi) d \xi \\
-\int_{z}^{\infty} e^{B_{\infty} P^{c u}(z-\xi)} P^{c u} L(\xi ; A) U(\xi) d \xi .
\end{gathered}
$$

(ii) if $U$ is bounded on $\mathbb{R}_{-}$, then for every $R \geq 0$ there exist $a V_{0}^{u} \in X^{u}$ and $a V_{0}^{c} \in X^{c}$ such that for $z \leq-R$

$$
\begin{gathered}
U(z)=e^{B_{\infty} P^{u}(z+R)} V_{0}^{u}+e^{B_{\infty} P^{c} z} V_{0}^{c}-\int_{z}^{-R} e^{B_{\infty} P^{u}(z-\xi)} P^{u} L(\xi ; A) U(\xi) d \xi \\
\quad+\int_{-\infty}^{z} e^{B_{\infty} P^{c s}(z-\xi)} P^{c s} L(\xi ; A) U(\xi) d \xi .
\end{gathered}
$$


Proof. Define $M: L^{2}\left(S^{1}\right) \times L^{2}\left(S^{1}\right)=: \widetilde{X} \rightarrow X$ by

$$
M U(\varphi)=\sum_{k \in \mathbb{Z}} M_{k} \widehat{U}_{k} e^{i k \varphi},
$$

where $M_{k}$ is defined as in (6), and $\widehat{U}_{k}$ are the Fourier coefficients of $U$ as before. Note that $M$ is a linear homeomorphism. Define also the unbounded operator $D(\lambda)$ on $\widetilde{X}$ by

$$
D U(\varphi)=\sum_{k \in \mathbb{Z}} D_{k} \widehat{U}_{k} e^{i k \varphi}
$$

where $D_{k}$ is defined in (7). Note that $D$ is closed and densely defined with domain $H^{1}\left(S^{1}\right) \times$ $H^{1}\left(S^{1}\right)$.

After the change of variables $U=M \widetilde{U}$, the system (3) can be rewritten as

$$
\widetilde{U}^{\prime}=D \widetilde{U}+\widetilde{L}(z ; A) \widetilde{U},
$$

where $\widetilde{L}(z ; A):=M^{-1} L(z ; A) M$. Then $U \in X$ if and only if $\widetilde{U} \in \widetilde{X}$. Note that $\widetilde{L}$ has the same decay properties as $L$. It follows from the definition of $X_{\beta}$ that

$$
\sup _{z \in \mathbb{R}}(1+|z|)^{\beta}\|L(z ; A)\|_{\mathcal{L}(X)}<\infty
$$

and so we see that

$$
\sup _{z \in \mathbb{R}}(1+|z|)^{\beta}\|\widetilde{L}(z ; A)\|_{\mathcal{L}(\widetilde{X})}<\infty .
$$

Hence we introduce the space $\widetilde{X}_{\beta}$ with norm defined by

$$
\|\widetilde{L}\|_{\widetilde{X}_{\beta}}=\sup _{z \in \mathbb{R}}(1+|z|)^{\beta}\|\widetilde{L}(z ; A)\|_{\widetilde{\mathcal{L}}(X)}
$$

In the rest of the proof we use the notation $\widetilde{P}^{s}:=M^{-1} P^{s}, \widetilde{P}^{u}:=M^{-1} P^{u}, \widetilde{P}^{c}:=M^{-1} P^{c}$, and $\widetilde{X}^{s}:=\operatorname{Ran} \widetilde{P}^{s}$, etc.

We only consider the case when $U$ is bounded on $\mathbb{R}_{+}$, since the case for $\mathbb{R}_{-}$is completely similar. We introduce $\widetilde{U}_{j}(z):=\widetilde{P}^{j} U(z)$, for $j \in\{s, c, u\}$. By the variation of constants formula (see e.g. [15]), it follows after projecting onto $\widetilde{X}^{s}, \widetilde{X}^{c}$, and $\widetilde{X}^{u}$, respectively, that any solution of (13) satisfies

$$
\begin{aligned}
& \widetilde{U}^{s}(z)=e^{D \widetilde{P}^{s}(z-\zeta)} \widetilde{U}^{s}(\zeta)+\int_{\zeta}^{z} e^{D \widetilde{P}^{s}(z-\xi)} \widetilde{P}^{s} \widetilde{L}(\xi ; A) \widetilde{U}(\xi) d \xi, \quad z \geq \zeta \geq 0, \\
& \widetilde{U}^{c}(z)=e^{D \widetilde{P}^{c}(z-\zeta)} \widetilde{U}^{c}(\zeta)+\int_{\zeta}^{z} e^{D \widetilde{P}^{c}(z-\xi)} \widetilde{P}^{c} \widetilde{L}(\xi ; A) \widetilde{U}(\xi) d \xi, \quad z, \zeta \geq 0, \\
& \widetilde{U}^{u}(z)=e^{D \widetilde{P}^{u}(z-\zeta)} \widetilde{U}^{u}(\zeta)+\int_{\zeta}^{z} e^{D \widetilde{P}^{u}(z-\xi)} \widetilde{P} u \widetilde{L}(\xi ; A) \widetilde{U}(\xi) d \xi, \quad \zeta \geq z \geq 0,
\end{aligned}
$$

Since $\|U(z)\|_{X}$ is bounded, also $\|\widetilde{U}(z)\|_{\tilde{X}}$ is bounded as $z \rightarrow+\infty$, and therefore $\widetilde{U}^{s}, \widetilde{U}^{c}$ and $\widetilde{U}^{u}$ are all bounded.

We first look at $\widetilde{U}^{u}$ and let $\zeta \rightarrow+\infty$ in the last equation of (14). Since $\widetilde{U}^{u}$ is bounded, it follows that

$$
\widetilde{U}^{u}(z)=-\int_{z}^{\infty} e^{D \widetilde{P}^{u}(z-\xi)} \widetilde{P}^{u} \widetilde{L}(\xi ; A) \widetilde{U}(\xi) d \xi
$$

Next, we study the equation for $\widetilde{U}^{c}$. The integral on the right hand side of the second equation of (14) converges as $\zeta \rightarrow+\infty$ since $\left\|e^{D P^{c} \xi}\right\|$ is uniformly bounded for $\xi \in \mathbb{R}$ and since

$$
\begin{aligned}
\int_{z}^{\infty}\|\widetilde{L}(\xi ; A) \widetilde{U}(\xi)\|_{\widetilde{X}} d \xi & \leq\|\widetilde{L}(\cdot ; A)\|_{\widetilde{X}_{\beta}}\|\widetilde{U}\|_{L^{\infty}} \int_{z}^{\infty} \frac{1}{(1+\xi)^{\beta}} d \xi \\
& =\frac{1}{\beta-1}\|\widetilde{L}(\cdot ; A)\|_{\widetilde{X}_{\beta}}\|\widetilde{U}\|_{L^{\infty}} \frac{1}{(1+z)^{\beta-1}},
\end{aligned}
$$


where we recall that $\beta>1$. Since the left hand side of the second equation in (14) does not depend

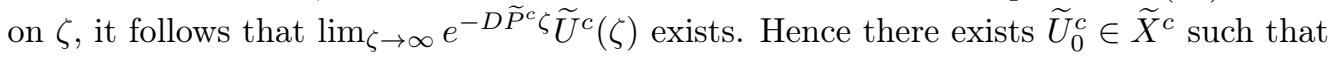

$$
\widetilde{U}^{c}(z)=e^{D \widetilde{P}^{c} z} \widetilde{U}_{0}^{c}-\int_{z}^{\infty} e^{D \widetilde{P}^{c}(z-\xi)} \widetilde{P}^{c} \widetilde{L}(\xi ; A) \widetilde{U}(\xi) d \xi .
$$

For $\widetilde{U}^{s}$, we choose $\zeta=R \geq 0$ arbitrarily, so that by (14) for $z \geq R$

$$
\begin{gathered}
\widetilde{U}(z)=e^{D \widetilde{P}^{s}(z-R)} \widetilde{U}^{s}(R)+e^{D \widetilde{P}^{c} z} \widetilde{U}_{0}^{c}+\int_{R}^{z} e^{D \widetilde{P}^{s}(z-\xi)} \widetilde{P}^{s} \widetilde{L}(\xi ; A) \widetilde{U}(\xi) d \xi \\
-\int_{z}^{\infty} e^{D \widetilde{P}^{c u}(z-\xi)} \widetilde{P}^{c u} \widetilde{L}(\xi ; A) \widetilde{U}(\xi) d \xi .
\end{gathered}
$$

Finally, we write $\widetilde{U}_{0}^{s}=\widetilde{U}^{s}(R)$ and transform back to the $U$ variable. By putting $U_{0}^{s}:=M \widetilde{U}_{0}^{s}$, etc. we obtain the expression in the lemma.

We now use the expression of Lemma 5 and use a contraction mapping argument to show that any eigenfunction is exponentially decaying.

Lemma 6. Let $A$ be a fixed function which satisfies (A1) and (A2), and assume that $\lambda$ is an eigenvalue of the perturbed operator $\mathcal{L}_{A}$ with eigenvalue $\lambda>0$ and corresponding eigenfunction $u \in H^{2}\left(\mathbb{R} \times S^{1}\right)$. We assume that $\sqrt{\lambda} \notin \mathbb{Z}$. We denote by $U$ the corresponding solution of the dynamical system (3). Let $\tilde{\kappa} \in\left(0, \mu_{\min }(\lambda)\right)$. Then there exists a constant $K>0$ such that

$$
\|U(z)\|_{X} \leq K e^{-\tilde{\kappa}|z|}
$$

for all $z \in \mathbb{R}$.

Proof. Since $u \in H^{2}\left(\mathbb{R} \times S^{1}\right)$ and $U=\left(u, u^{\prime}\right)^{T}$, it follows that $U \in H^{1}(\mathbb{R} ; X)$. By standard arguments, $\|U(z)\|_{X} \rightarrow 0$ as $|z| \rightarrow \infty$. We concentrate on the proof for $z \rightarrow+\infty$ since the argument for $z \rightarrow-\infty$ is completely similar. We estimate the integrals in (11) and use the notation $L(z)$ in place of $L(z ; A)$ :

$$
I_{1}=\int_{R}^{z} e^{B_{\infty} P^{s}(z-\xi)} P^{s} L(\xi) U(\xi) d \xi \text { and } I_{2}=\int_{z}^{\infty} e^{B_{\infty} P^{c u}(z-\xi)} P^{c u} L(\xi) U(\xi) d \xi .
$$

By (15) and since $\left\|e^{B \infty} P^{c u} \tau P^{c u}\right\|_{\mathcal{L}(X)} \leq 1$ for any $\tau<0$, it follows that

$$
\left\|I_{2}\right\|_{X} \leq \frac{1}{\beta-1}\|L\|_{X_{\beta}}\|U\|_{L^{\infty}} \frac{1}{(1+z)^{\beta-1}} .
$$

To estimate $I_{1}$, we use that for any $\alpha \in \mathbb{R}$

$$
\lim _{r \rightarrow \infty} \int_{1}^{r}\left(\frac{r}{s}\right)^{\alpha} e^{-(r-s)} d s=1
$$

which implies that there exists a constant $C>0$ such that for every $r \geq 1$,

$$
\int_{1}^{r}\left(\frac{r}{s}\right)^{\alpha} e^{-(r-s)} d s \leq C .
$$

Let $\kappa=\mu_{\min (\lambda)}$. By the substitution $\kappa(1+\xi)=\tau$ and equation (16) we have for $z \geq R$

$$
\begin{aligned}
\left\|I_{1}\right\|_{X} & \leq \frac{\|L\|_{X_{\beta}}\|U\|_{L^{\infty}}}{(1+z)^{\beta}} \int_{R}^{z} e^{-\kappa(z-\xi)}\left(\frac{1+z}{1+\xi}\right)^{\beta} d \xi \\
& =\frac{\|L\|_{X_{\beta}}\|U\|_{L^{\infty}}}{\kappa(1+z)^{\beta}} \int_{\kappa(1+R)}^{\kappa(1+z)}\left(\frac{\kappa(1+z)}{\tau}\right)^{\beta} e^{-(\kappa(1+z)-\tau)} d \tau \\
& \leq C \frac{\|L\|_{X_{\beta}}\|U\|_{L^{\infty}}}{\kappa(1+z)^{\beta}}
\end{aligned}
$$

provided that $\kappa(1+R) \geq 1$, which in particular holds if $R \geq 1 / \kappa$. This shows that $I_{1}$ and $I_{2}$ converge to 0 as $z \rightarrow \infty$. Then by (11) it follows that $U(z) \rightarrow U_{0}^{c}$ as $z \rightarrow \infty$. Since $U(z) \rightarrow 0$ as $z \rightarrow \infty$, we must have $U_{0}^{c}=0$. 
Hence for $z \geq R \geq 1 / \kappa, U(z)$ satisfies

$$
\begin{gathered}
U(z)=e^{B_{\infty} P^{s}(z-R)} U^{s}(R)+\int_{R}^{z} e^{B_{\infty} P^{s}(z-\xi)} P^{s} L(\xi) U(\xi) d \xi \\
-\int_{z}^{\infty} e^{B_{\infty} P^{c u}(z-\xi)} P^{c u} L(\xi) U(\xi) d \xi .
\end{gathered}
$$

For $R \geq 1 / \kappa$ fixed and $\eta \geq 0$ we define the spaces

$$
Y_{\eta}=\left\{W \in C([R, \infty) ; \mathcal{L}(X)) ;\|W\|_{Y_{\eta}}:=\sup _{z \geq R} e^{\eta z}\|W(z)\|_{X}<\infty\right\} .
$$

Then for $\eta \in[0, \kappa)$ we let $F: Y_{\eta} \rightarrow Y_{\eta}$ be given by

$$
\begin{gathered}
F(W)(z)=e^{B_{\infty} P^{s}(z-R)} U^{s}(R)+\int_{R}^{z} e^{B_{\infty} P^{s}(z-\xi)} P^{s} L(\xi) W(\xi) d \xi \\
-\int_{z}^{\infty} e^{B_{\infty} P^{c u}(z-\xi)} P^{c u} L(\xi) W(\xi) d \xi
\end{gathered}
$$

To see that the right hand side belongs to $Y_{\eta}$, note that $P^{s} U^{s}=U^{s}$ and that $e^{B_{\infty} P^{s} \tau} P^{s}$ is exponentially decaying with rate $e^{-\kappa \tau}$ for $\tau \rightarrow \infty$, and so $e^{B \infty P^{s}(z-R)} U^{s}(R) \in Y_{\eta}$. To see that the integrals belong to $Y_{\eta}$, similar arguments as those below can be used.

Let $W_{1}, W_{2} \in Y_{\eta}$. It follows that

$$
\begin{gathered}
\left\|F\left(W_{1}\right)-F\left(W_{2}\right)\right\|_{Y_{\eta}} \leq\left\|W_{1}-W_{2}\right\|_{Y_{\eta}}\left(\sup _{z \geq R} \int_{R}^{z}\left\|e^{B \infty P^{s}(z-\xi)} P^{s}\right\|_{\mathcal{L}(X)}\|L(\xi)\|_{\mathcal{L}(X)} e^{\eta(z-\xi)} d \xi\right. \\
\left.\quad+\sup _{z \geq R} \int_{z}^{\infty}\left\|e^{B_{\infty} P^{c u}(z-\xi)} P^{c u}\right\|_{\mathcal{L}(X)}\|L(\xi)\|_{\mathcal{L}(X)} e^{\eta(z-\xi)} d \xi\right) \\
\leq K\|L\|_{X_{\beta}}\left\|W_{1}-W_{2}\right\|_{Y_{\eta}}\left(\sup _{z \geq R} \frac{1}{(1+z)^{\beta}} \int_{R}^{z} e^{-(\kappa-\eta)(z-\xi)}\left(\frac{1+z}{1+\xi}\right)^{\beta} d \xi\right. \\
\left.\quad+\sup _{z \geq R} \int_{z}^{\infty} \frac{1}{(1+\xi)^{\beta}} d \xi\right) \\
\leq K\|L\|_{X_{\beta}}\left\|W_{1}-W_{2}\right\|_{Y_{\eta}}\left(\frac{C}{(\kappa-\eta)(1+R)^{\beta}}+\frac{1}{(\beta-1)(1+R)^{\beta-1}}\right) .
\end{gathered}
$$

By choosing $R$ large enough, we see that $F$ is a contraction on $Y_{\eta}$. Hence $F$ has a unique fixed point in $Y_{\eta}$ for any $\eta \in[0, \kappa)$.

Since $U \in Y_{0}$ solves (17), it is a fixed point for $F$ when $\eta=0$. But $Y_{\eta} \subset Y_{0}$ for $\eta>0$, so by uniquenss of the fixed point in $Y_{0}$, these fixed points must be the same. Hence $U \in Y_{\eta}$ for any $\eta \in[0, \kappa)$. This shows that $U$ decays exponentially.

\section{LyAPUNOV-SCHMidT REDUCTION}

We are now ready to prove our main result. For this we use Lyapunov-Schmidt reduction. Let $u_{*}$ be the eigenfunction associated with the embedded eigenvalue of the unperturbed problem, i.e. $\mathcal{L}_{A_{0}} u_{*}=\lambda_{0} u_{*}$. We may without loss of generality assume that $u_{*}$ is normalized so that $\int_{-\infty}^{\infty}\left\|u_{*}(z)\right\|_{L^{2}\left(S^{1}\right)}^{2} d z=1$. We write $U_{*}:=\left(u_{*}, u_{*}^{\prime}\right)^{T}$, i.e. $U_{*}$ is a solution of $(3)$ with $A=A_{0}$ and $\lambda=\lambda_{0}$.

Using the evolution operators and related projections defined in (10), we define the stable and unstable subspaces $E_{+}^{s}$ and $E_{-}^{u}$ by

$$
\begin{aligned}
& E_{+}^{s}=\left\{U \in Y ; P_{+}^{s}\left(0 ; \lambda_{0}, A_{0}\right) U=U\right\}, \\
& E_{-}^{u}=\left\{U \in Y ; P_{-}^{u}\left(0 ; \lambda_{0}, A_{0}\right) U=U\right\} .
\end{aligned}
$$

Roughly speaking, $E_{+}^{s}$ and $E_{-}^{u}$ consist of the initial values of solutions of the unperturbed system which decay exponentially as $z \rightarrow+\infty$ and $z \rightarrow-\infty$, respectively. We have $E_{+}^{s} \cap E_{-}^{u}=$ $\operatorname{span}\left\{U_{*}(0)\right\}$ since $\lambda_{0}$ is an eigenvalue with multiplicity 1 . The following is a corollary of Lemma 6. 
Corollary 2. Let $U$ be a solution of (3). If $U(0) \in E_{+}^{s} \cap E_{-}^{u}$, then $U_{0}^{c}=V_{0}^{c}=0$ in equation (11) and (12).

To find embedded eigenvalues, we define the mapping $\iota: E_{+}^{s} \times E_{-}^{u} \times \mathbb{R} \times X_{\beta} \rightarrow X$ by

$$
\iota:\left(U_{0}^{s}, U_{0}^{u} ; \lambda, A\right)=P_{+}^{s}(0 ; \lambda, A) U_{0}^{s}-P_{-}^{u}(0 ; \lambda, A) U_{0}^{u} .
$$

Lemma 7. $\lambda \in\left((m-1)^{2}, m^{2}\right)$ is an eigenvalue of $\mathcal{L}_{A}$ if and only if there exist $U_{0}^{s} \in E_{+}^{s}$ and $U_{0}^{u} \in E_{-}^{u}$ with $\left(U_{0}^{s}, U_{0}^{u}\right) \neq(0,0)$ such that

$$
\iota\left(U_{0}^{s}, U_{0}^{u} ; \lambda, A\right)=0 .
$$

Proof. If (18) holds, then $P_{+}^{s}(0 ; \lambda, A) U_{0}^{s}=P_{-}^{u}(0 ; \lambda, A) U_{0}^{u}$, which implies that the solution of (3) with initial value $P_{+}^{s}(0 ; \lambda, A) U_{0}^{s}=P_{-}^{u}(0 ; \lambda, A) U_{0}^{u}$ decays exponentially as $|z| \rightarrow \infty$. By Lemma 1 , it follows that $\lambda$ is an eigenvalue of the perturbed operator $\mathcal{L}_{A}$.

Conversely, if $\lambda$ is an eigenvalue, then Lemma 6 guarantees that (3) has a solution $U$ which decays exponentially as $|z| \rightarrow \infty$ with rate at least $\widetilde{\kappa}$. Thus, $U(0)=P_{+}^{s}(0 ; \lambda, A) U(0)$ and $U(0)=$ $P_{-}^{u}(0 ; \lambda, A) U(0)$, which means that (18) holds.

To solve (18), first note that for any $\left(U_{0}^{s}, U_{0}^{u}\right) \in E_{+}^{s} \times E_{-}^{u}$ we have

$$
\iota\left(U_{0}^{s}, U_{0}^{u} ; \lambda_{0}, A_{0}\right)=U_{0}^{s}-U_{0}^{u} .
$$

Hence $\operatorname{Ran} \iota\left(\cdot, \cdot ; \lambda_{0}, A_{0}\right)=E_{+}^{s}+E_{-}^{u}$. Next we will show that the codimension of $E_{+}^{s}+E_{-}^{u}$ is one higher than the dimension of $X^{c}$.

Lemma 8. We have $\operatorname{codim}\left(E_{+}^{s}+E_{-}^{u}\right)=\operatorname{dim}\left(X^{c}\right)+1=4 m-1$.

Proof. We show below that $\iota\left(\cdot, \cdot ; \lambda_{0}, A_{0}\right)$ is Fredholm with Fredholm index

$$
\operatorname{ind}(\iota)=-\operatorname{dim}\left(X^{c}\right)=-(4 m-2) \text {. }
$$

It then follows from $\operatorname{Ran} \iota\left(\cdot, \cdot ; \lambda_{0}, A_{0}\right)=E_{+}^{s}+E_{-}^{u}$ and the definition of Fredholm index that

$$
\begin{aligned}
\operatorname{codim}\left(E_{+}^{s}+E_{-}^{u}\right) & =\operatorname{dim}\left(\operatorname{ker} \iota\left(\cdot, \cdot ; \lambda_{0}, A_{0}\right)\right)-\operatorname{ind}\left(\iota\left(\cdot, \cdot ; \lambda_{0}, A_{0}\right)\right) \\
& =1-(-(4 m-2))=4 m-1 .
\end{aligned}
$$

We have checked in Lemma 4 that the conditions of Theorem 1 of [20] are satisfied. Hence, by the same theorem, for any $\epsilon>0$ there exist operators $S_{1}, S_{2}, K_{1}$ and $K_{2}$ in $\mathcal{L}\left(E_{+}^{s} \times E_{-}^{u}, X\right)$ with $\left\|S_{j}\right\|_{\mathcal{L}\left(E_{+}^{s} \times E_{-}^{u}, X\right)} \leq \epsilon / 2$ and $K_{j}$ compact for $j=1,2$ and such that

$$
\begin{aligned}
& \iota\left(U_{0}^{s}, U_{0}^{u} ; \lambda_{0}, A_{0}\right)=U_{0}^{s}-U_{0}^{u} \\
& \quad=P^{s}\left(\lambda_{0}\right) U_{0}^{s}-P^{u}\left(\lambda_{0}\right) U_{0}^{u}+\left(I-P^{s}\left(\lambda_{0}\right)\right)\left(S_{1}+K_{1}\right) U_{0}^{s}-\left(I-P^{u}\left(\lambda_{0}\right)\right)\left(S_{2}+K_{2}\right) U_{0}^{u} .
\end{aligned}
$$

We will prove that $\iota$ is Fredholm by writing

$$
\iota\left(U_{0}^{s}, U_{0}^{u} ; \lambda_{0}, A_{0}\right)=\tilde{\iota}\left(U_{0}^{s}, U_{0}^{u}\right)+(S+K)\left(U_{0}^{s}, U_{0}^{u}\right),
$$

where $\tilde{\iota}: E_{+}^{s} \times E_{-}^{u} \rightarrow X$ is Fredholm with index $-(4 m-2)$ and $\|S\|_{\mathcal{L}\left(E_{+}^{s} \times E_{-}^{u}, X\right)} \leq \epsilon$ and $K$ : $E_{+}^{s} \times E_{-}^{u} \rightarrow X$ is compact. Indeed, let

$$
\begin{aligned}
\tilde{\iota}\left(U_{0}^{s}, U_{0}^{u}\right) & =P^{s}\left(\lambda_{0}\right) U_{0}^{s}-P^{u}\left(\lambda_{0}\right) U_{0}^{u}, \\
S\left(U_{0}^{s}, U_{0}^{u}\right) & =\left(I-P^{s}\left(\lambda_{0}\right)\right) S_{1} U_{0}^{s}-\left(I-P^{u}\left(\lambda_{0}\right)\right) S_{2} U_{0}^{u}, \\
K\left(U_{0}^{s}, U_{0}^{u}\right) & =\left(I-P^{s}\left(\lambda_{0}\right)\right) K_{1} U_{0}^{s}-\left(I-P^{u}\left(\lambda_{0}\right)\right) K_{2} U_{0}^{u} .
\end{aligned}
$$

By Lemma 3.3 of $[20]$ the operators $P^{s}\left(\lambda_{0}\right): E_{+}^{s} \rightarrow X^{s}$ and $P^{u}\left(\lambda_{0}\right): E_{-}^{u} \rightarrow X^{u}$ are both Fredholm with index 0. Since $X^{u} \cap X^{s}=\{0\}$, it follows that $\operatorname{ker} \tilde{\iota}=\left.\operatorname{ker} P^{s}\right|_{E_{+}^{s}} \times\left.\operatorname{ker} P^{u}\right|_{E_{-}^{u}}$, so that $\operatorname{dim} \operatorname{ker} \tilde{\iota}=\left.\operatorname{dim} \operatorname{ker} P^{s}\right|_{E_{+}^{s}}+\left.\operatorname{dim} \operatorname{ker} P^{u}\right|_{E_{-}^{u}}$. Moreover, $\operatorname{Ran} \tilde{\iota}=\left.\left.\operatorname{Ran} P^{s}\right|_{E_{+}^{s}} \oplus \operatorname{Ran} P^{u}\right|_{E_{-}^{u}}$, and so $\operatorname{dim} \operatorname{Ran} \tilde{\iota}=\left.\operatorname{dim} \operatorname{Ran} P^{s}\right|_{E_{+}^{s}}+\left.\operatorname{dim} \operatorname{Ran} P^{u}\right|_{E_{-}^{u}}$. It follows that $\iota: E_{+}^{s} \times E_{-}^{u} \rightarrow X^{s} \oplus X^{u}$ is Fredholm with Fredholm index 0 . When considering $\tilde{\iota}$ as an operator into the bigger space $X=X^{s} \oplus X^{u} \oplus X^{c}$, the codimension of the range will increase by the number of dimensions of $X^{c}$, i.e. $4 m-2$, while the dimension of the kernel is unchanged. By the definition of Fredholm index, the index of $\tilde{\iota}: E_{+}^{s} \times E_{-}^{u} \rightarrow X$ is then $-(4 m-2)$. 
It is straight-forward to prove that $\|S\|_{\mathcal{L}\left(E_{+}^{s} \times E_{-}^{u}, X\right)} \leq \epsilon$ :

$$
\begin{aligned}
\left\|S\left(U_{0}^{s}, U_{0}^{u}\right)\right\| & \leq\left\|S_{1} U_{0}^{s}\right\|+\left\|S_{2} U_{0}^{u}\right\| \\
& \leq\left\|S_{1}\right\|\left\|U_{0}^{s}\right\|+\left\|S_{2}\right\|\left\|U_{0}^{u}\right\| \\
& \leq \frac{\epsilon}{2}\left(\left\|U_{0}^{s}\right\|+\left\|U_{0}^{u}\right\|\right) \\
& \leq \epsilon \sqrt{\left\|U_{0}^{s}\right\|^{2}+\left\|U_{0}^{u}\right\|^{2}},
\end{aligned}
$$

which proves that $\|S\| \leq \epsilon$.

It is also easy to see that $K$ is compact, using that $K_{1}$ and $K_{2}$ are compact. Since $\epsilon$ can be made arbitrarily small, it follows from Fredholm theory (see e.g. Theorem 5.22 and Theorem 5.26 of Chapter IV of [16]) that $\iota: E_{+}^{s} \times E_{-}^{u} \rightarrow X$ is also Fredholm with index $-(4 m-2)$. This completes the proof.

Let $Q$ be a projection in $X$ onto $\operatorname{Ran} \iota\left(\cdot, \cdot ; \lambda_{0}, A_{0}\right)=E_{+}^{s}+E_{-}^{u}$. Lemma 8 implies that $\operatorname{dim}(\operatorname{ker} Q)=4 m-1$. Equation (18) can then be rewritten as

$$
\begin{aligned}
Q \iota\left(U_{0}^{s}, U_{0}^{u} ; \lambda, A\right) & =0, \\
(I-Q) \iota\left(U_{0}^{s}, U_{0}^{u} ; \lambda, A\right) & =0 .
\end{aligned}
$$

Lemma 9. Let $D \subset E_{+}^{s} \times E_{-}^{u}$ be an affine hyperplane such that

$$
D \cap \operatorname{span}\left\{\left(U_{*}(0), U_{*}(0)\right)\right\}=\left\{\left(U_{*}(0), U_{*}(0)\right)\right\} .
$$

For $(\lambda, A)$ close to $\left(\lambda_{0}, A_{0}\right)$, the first equation of (20) has a unique solution

$$
\left(U_{0}^{s}, U_{0}^{u}\right)=\left(U_{0}^{s}(\lambda, A), U_{0}^{u}(\lambda, A)\right) \in D
$$

in a neighbourhood of $\left(U_{*}(0), U_{*}(0)\right)$. Moreover, $U_{0}^{s}$ and $U_{0}^{u}$ are smooth in their arguments.

Proof. For $(\lambda, A)$ fixed, $Q \iota$ is a linear mapping from $E_{+}^{s} \times E_{-}^{u}$ to $\operatorname{Ran} Q=E_{+}^{s}+E_{-}^{u}$, and $\operatorname{ker}(Q \iota)\left(\cdot, \cdot ; \lambda_{0}, A_{0}\right)=\operatorname{span}\left\{\left(U_{*}(0), U_{*}(0)\right)\right\}$. By the definition of $Q$ and since $P_{+}^{s}$ and $P_{-}^{u}$ are continuous in $\lambda$ and $A$, it follows that $Q \iota(\cdot, \cdot ; \lambda, A): E_{+}^{s} \times E_{-}^{u} \rightarrow E_{+}^{s}+E_{-}^{u}$ is surjective for $(\lambda, A)$ in a neighbourhood of $\left(\lambda_{0}, A_{0}\right)$.

The implicit function theorem now gives that for $(\lambda, A)$ close to $\left(\lambda_{0}, A_{0}\right)$, the first equation of (20) has a unique solution $\left(U_{0}^{s}(\lambda, A), U_{0}^{u}(\lambda, A)\right) \in D$ in a neighbourhood of $\left(U_{*}(0), U_{*}(0)\right)$. It also follows that this solution is smooth in its arguments.

Using the variation of constants formula (see [15]), it is easy to see that

$$
\begin{aligned}
\iota(\cdot, \cdot ; \lambda, A)=U_{0}^{s} & -U_{0}^{u}+\int_{-\infty}^{0} \Phi_{-}^{c s}\left(0, z ; \lambda, A_{0}\right) L(z ; A) \Phi_{-}^{u}(z, 0 ; \lambda, A) U_{0}^{u} d z \\
& +\int_{0}^{\infty} \Phi_{+}^{c u}\left(0, z ; \lambda, A_{0}\right) L(z ; A) \Phi_{+}^{s}(z, 0 ; \lambda, A) U_{0}^{s} d z .
\end{aligned}
$$

To solve the second equation of (20), we define $F: \mathbb{R} \times X_{\beta} \rightarrow \operatorname{ker} Q$ by

$$
\begin{aligned}
F(\lambda, A) & =-\iota\left(U_{0}^{s}(\lambda, A), U_{0}^{u}(\lambda, A) ; \lambda, A\right)=-(I-Q) \iota\left(U_{0}^{s}(\lambda, A), U_{0}^{u}(\lambda, A)\right) \\
& =\int_{-\infty}^{0}(I-Q) \Phi_{-}^{c s}\left(0, z ; \lambda_{0}, A_{0}\right)\left(\left(\lambda-\lambda_{0}\right) N+L(z ; A)\right) \Phi_{-}^{u}(z, 0 ; \lambda, A) d z \\
& +\int_{0}^{\infty}(I-Q) \Phi_{+}^{c u}\left(0, z ; \lambda_{0}, A_{0}\right)\left(\left(\lambda-\lambda_{0}\right) N+L(z ; A)\right) \Phi_{+}^{s}(z, 0 ; \lambda, A) d z,
\end{aligned}
$$

where $N: X \rightarrow X$ is given by

$$
N=\left(\begin{array}{ll}
0 & 0 \\
1 & 0
\end{array}\right) .
$$

Note that $F$ is a smooth function and that solving (20) is equivalent to solving $F(\lambda, A)=0$.

To solve $F(\lambda, A)=0$, we use the dual space $X^{\prime}=H^{-1}\left(S^{1}\right) \times L^{2}\left(S^{1}\right)$ of $X$ and the adjoint equation for $\lambda=\lambda_{0}$ and $A=A_{0}$, i.e. the equation

$$
W^{\prime}=-\left(B_{\infty}\left(\lambda_{0}\right)+L\left(z ; A_{0}\right)\right)^{*} W .
$$


The adjoint system has exponential dichotomies on $\mathbb{R}_{+}$and $\mathbb{R}_{-}$which we denote by $\Psi_{+}^{s}\left(z, z_{0}\right)$, $\Psi_{+}^{c u}\left(z, z_{0}\right)$ and $\Psi_{-}^{u}\left(z, z_{0}\right), \Psi_{-}^{c s}\left(z, z_{0}\right)$, respectively. The relationships between the dichotomies for the unperturbed system (3) with $\lambda=\lambda_{0}$ and $A=A_{0}$ and those of the adjoint system (22) are given by $\Psi_{+}^{s}\left(z, z_{0}\right)=\Phi_{+}^{c u}\left(z_{0}, z\right)^{*}, \Psi_{+}^{c u}\left(z, z_{0}\right)=\Phi_{+}^{s}\left(z_{0}, z\right)^{*}$, etc., where $z_{0}, z$ are in the appropriate intervals.

It is straight forward to check that $U_{*}^{\perp}:=\left(-u_{*}^{\prime}, u_{*}\right)^{T}$ solves the adjoint system $(22)$ and is exponentially decaying as $|z| \rightarrow \infty$. It is also easy to see that for $U^{s} \in E_{+}^{s}$ and $U^{u} \in E_{-}^{u}$ we have

$$
\begin{aligned}
\frac{d}{d z}\left\langle U_{*}^{\perp}(z), \Phi_{+}^{s}(z, 0) U^{s}\right\rangle & =\frac{d}{d z}\left\langle\Psi_{+}^{s}(z, 0) U_{*}^{\perp}(0), \Phi_{+}^{s}(z, 0) U^{s}\right\rangle=0, \\
\frac{d}{d z}\left\langle U_{*}^{\perp}(z), \Phi_{-}^{u}(z, 0) U^{u}\right\rangle & =\frac{d}{d z}\left\langle\Psi_{-}^{u}(z, 0) U_{*}^{\perp}(0), \Phi_{-}^{u}(z, 0) U^{u}\right\rangle=0,
\end{aligned}
$$

and since $U_{*}^{\perp}(z) \rightarrow 0$ as $|z| \rightarrow \infty$ and $\Phi_{+}^{s}(z, 0) U^{s}$ and $\Phi_{-}^{u}(z, 0) U^{u}$ are bounded on $\mathbb{R}_{+}$and $\mathbb{R}_{-}$, respectively, it follows that $\left\langle U_{*}^{\perp}(0), U^{s}+U^{u}\right\rangle=0$. This also implies that for any $U \in X$ we have $\left\langle U_{*}^{\perp}(0), Q U\right\rangle=0$, and thus also $\left\langle Q^{*} U_{*}^{\perp}(0), U\right\rangle=0$. This gives immediately that $U_{*}^{\perp}(0) \in \operatorname{ker} Q^{*}$.

Lemma 10. The equation $\left\langle U_{*}^{\perp}(0), F(\lambda, A)\right\rangle=0$ defines a smooth function $\lambda(A)$ in a neighbourhood of $A=A_{0}$ such that $\lambda\left(A_{0}\right)=\lambda_{0}$. Furthermore, for any $A \in X_{\beta}$,

$$
\lambda^{\prime}\left(A_{0}\right) A=-\int_{-\infty}^{\infty}\left(u_{*}(z),\left(-2 i A(z, \cdot) \partial-i A_{\varphi}^{\prime}(z, \cdot)+2 A_{0}(z, \cdot) A(z, \cdot)\right) u_{*}(z)\right)_{L^{2}\left(S^{1}\right)} d z .
$$

Proof. The pairing of $F(\lambda, A)$ with $U_{*}^{\perp}(0)$ gives the equation

$$
\begin{aligned}
0 & =F_{*}(\lambda, A):=\left\langle U_{*}^{\perp}(0), F(\lambda, A)\right\rangle \\
& =\int_{-\infty}^{0}\left\langle U_{*}^{\perp}(0), \Phi_{-}^{c s}\left(0, z ; \lambda_{0}, A_{0}\right)\left(\left(\lambda-\lambda_{0}\right) N+L(z ; A)\right) \Phi_{-}^{u}(z, 0 ; \lambda, A) U_{0}^{u}(\lambda, A)\right\rangle d z \\
& +\int_{0}^{\infty}\left\langle U_{*}^{\perp}(0), \Phi_{+}^{c u}\left(0, z ; \lambda_{0}, A_{0}\right)\left(\left(\lambda-\lambda_{0}\right) N+L(z ; A)\right) \Phi_{+}^{s}(z, 0 ; \lambda, A) U_{0}^{s}(\lambda, A)\right\rangle d z \\
& =\int_{-\infty}^{0}\left\langle U_{*}^{\perp}(z),\left(\left(\lambda-\lambda_{0}\right) N+L(z ; A)\right) \Phi_{-}^{u}(z, 0 ; \lambda, A) U_{0}^{u}(\lambda, A)\right\rangle d z \\
& +\int_{0}^{\infty}\left\langle U_{*}^{\perp}(z),\left(\left(\lambda-\lambda_{0}\right) N+L(z ; A)\right) \Phi_{+}^{s}(z, 0 ; \lambda, A) U_{0}^{s}(\lambda, A) d z .\right.
\end{aligned}
$$

By Lemma $4, F_{*}$ is a smooth function of $\lambda$ and $A$ in a neighbourhood of $\left(\lambda_{0}, A_{0}\right)$. Moreover, $F_{*}\left(\lambda_{0}, A_{0}\right)=0$ and

$$
\frac{\partial F_{*}}{\partial \lambda}\left(\lambda_{0}, A_{0}\right)=\int_{-\infty}^{\infty}\left\langle U_{*}^{\perp}(z), N U_{*}(z)\right\rangle d z=\int_{-\infty}^{\infty}\left\|u_{*}(z)\right\|_{L^{2}\left(S^{1}\right)}^{2} d z=1
$$

by the normalization of $u_{*}$.

This allows us to use the implicit function theorem to solve for $\lambda$ and obtain $\lambda$ as a function of $A$ in a neighbourhood of $A=A_{0}$ with $\lambda\left(A_{0}\right)=\lambda_{0}$. Differentiation of the function $F_{*}(\lambda(A), A)=0$ and evaluating in $A=A_{0}$ gives the expression (23).

We are now ready to prove Theorem 1.

Proof of Theorem 1. Since $\operatorname{ker} Q$ is $(4 m-1)$-dimensional, we have $4 m-2$ conditions left to verify. Again we use the adjoint equation. The adjoint projection $Q^{*}$ has a $(4 m-1)$-dimensional kernel, just as $Q$ itself. We have seen that $U_{*}^{\perp}(0) \in \operatorname{ker} Q$. Next, we define $W_{k}(0) \in X^{\prime}, k=$ $1, \ldots, 4 m-2$ to be such that $\left\{W_{k}(0) ; k=1, \ldots, 4 m-2\right\} \cup\left\{U_{*}^{\perp}(0)\right\}$ is a basis for $\operatorname{ker} Q^{*}$. For $k=1, \ldots, 4 m-2$, let $W_{k}$ be solution of the adjoint unperturbed system with initial value $W_{k}(0)$.

For $k=1, \ldots, 4 m-2$, we let

$$
F_{k}(A):=\left\langle W_{k}(0), F(\lambda(A), A)\right\rangle,
$$

and note that $F_{k}: X_{\beta} \rightarrow \mathbb{R}$ are smooth functions. If for some $A, F_{k}(A)=0$ for all $k=$ $1, \ldots, 4 m-2$, then $F(\lambda(A), A)=0$ as $\left\{W_{k}(0) ; k=1, \ldots, 4 m-2\right\} \cup\left\{U_{*}^{\perp}(0)\right\}$ is a basis for $\operatorname{ker} Q^{*}$. 
To prove the theorem, we show that there is a codimension $4 m-2$ manifold of perturbations $A$ defined by the equations $F_{k}(A)=0$ for $k=1, \ldots, 4 m-2$.

With the notation

$$
U(z ; A):= \begin{cases}\Phi_{+}^{s}(z, 0 ; \lambda(A), A) U_{0}^{s}(\lambda(A), A), & \text { for } z \geq 0 \\ \Phi_{-}^{u}(z, 0 ; \lambda(A), A) U_{0}^{u}(\lambda(A), A), & \text { for } z<0\end{cases}
$$

we have

$$
\begin{aligned}
F_{k}(A) & =\int_{-\infty}^{\infty}\left\langle W_{k}(z),\left(\left(\lambda(A)-\lambda_{0}\right) N+L(z ; A)\right) U(z ; A)\right\rangle d z \\
& =\int_{-\infty}^{\infty}\left(w_{k}(z),\left(\lambda(A)-\lambda_{0}-2 i A(z, \cdot) \partial-i A_{\varphi}^{\prime}(z, \cdot)+A(z, \cdot)^{2}\right) u(z ; A)\right)_{L^{2}\left(S^{1}\right)} d z
\end{aligned}
$$

where $W_{k}=\left(-w_{k}^{\prime}, w_{k}\right)^{T}$ and $u(z ; A)$ is the first component of $U(z ; A)$.

We claim that $F_{k}^{\prime}\left(A_{0}\right), k=1, \ldots, 4 m-2$ are linearly independent. To see this, we first observe that for every $A \in X_{\beta}$

$$
F_{k}^{\prime}\left(A_{0}\right) A=\int_{-\infty}^{\infty}\left(w_{k}(z),\left(\left[\lambda^{\prime}\left(A_{0}\right) A\right]-2 i A(z, \cdot) \partial-i A_{\varphi}^{\prime}(z, \cdot)+2 A_{0}(z, \cdot) A(z, \cdot)\right) u_{*}(z)\right)_{L^{2}\left(S^{1}\right)} d z
$$

where $\lambda^{\prime}\left(A_{0}\right) A$ is as in (23). Assume that there exist $\alpha_{1}, \ldots, \alpha_{4 m-2} \in \mathbb{C}$ such that for every $A \in X_{\beta}$

$$
\begin{aligned}
0 & =\sum_{k=1}^{4 m-2} \alpha_{k} F_{k}^{\prime}\left(A_{0}\right) A \\
& =\int_{-\infty}^{\infty}\left(w(z),\left(\left[\lambda^{\prime}\left(A_{0}\right) A\right]-2 i A(z, \cdot) \partial-i A_{\varphi}^{\prime}(z, \cdot)+2 A_{0}(z, \cdot) A(z, \cdot)\right) u_{*}(z)\right)_{L^{2}\left(S^{1}\right)} d z
\end{aligned}
$$

where we have used the notation

$$
w:=\sum_{k=1}^{4 m-2} \alpha_{k} w_{k}
$$

We will show that $\alpha_{k}=0$ for $k=1, \ldots, 4 m-2$. After a rearrangement and by (23), we see that this is equivalent to

$$
\int_{-\infty}^{\infty}\left(w(z)+\alpha_{*} u_{*}(z),\left(-2 i A(z, \cdot) \partial-i A_{\varphi}^{\prime}(z, \cdot)+2 A_{0}(z, \cdot) A(z, \cdot)\right) u_{*}(z)\right)_{L^{2}\left(S^{1}\right)} d z=0
$$

for every $A \in X_{\beta}$, where

$$
\alpha_{*}:=\int_{-\infty}^{\infty} \overline{\left(w(z), u_{*}(z)\right)_{L^{2}\left(S^{1}\right)}} d z
$$

Using integration by parts with respect to $\varphi$, it follows that

$$
\begin{aligned}
& \int_{-\infty}^{\infty} \int_{S^{1}}\left(i \frac{\partial}{\partial \varphi}\left(u_{*}(z, \varphi)\left(\overline{w(z, \varphi)+\alpha_{*} u_{*}(z, \varphi)}\right)\right)-2 i u_{* \varphi}^{\prime}(z, \varphi)\left(\overline{w(z, \varphi)+\alpha_{*} u_{*}(z, \varphi)}\right)\right. \\
& \left.\quad+2 A_{0}(z, \varphi) u_{*}(z, \varphi)\left(\overline{w(z, \varphi)+\alpha_{*} u_{*}(z, \varphi)}\right)\right) A(z, \varphi) d \varphi d z=0
\end{aligned}
$$

for every $A \in X_{\beta}$. Since $X_{\beta}$ is dense in $L^{2}\left(\mathbb{R} \times S^{1} ; \mathbb{R}\right)$, we get that

$i \frac{\partial}{\partial \varphi}\left(u_{*}(z, \varphi) \overline{\left(w+\alpha_{*} u_{*}\right)(z, \varphi)}\right)-2 i u_{* \varphi}^{\prime}(z, \varphi) \overline{\left(w+\alpha_{*} u_{*}\right)(z, \varphi)}+2 A_{0}(z, \varphi) u_{*}(z, \varphi) \overline{\left(w+\alpha_{*} u_{*}\right)(z, \varphi)}=0$.

After a simplification, it follows that

$$
u_{*}(z, \varphi) \overline{\left(w+\alpha_{*} u_{*}\right)_{\varphi}^{\prime}(z, \varphi)}-\left(u_{* \varphi}^{\prime}(z, \varphi)+2 i A_{0}(z, \varphi) u_{*}(z, \varphi)\right) \overline{\left(w+\alpha_{*} u_{*}\right)(z, \varphi)}=0
$$

for every $(z, \varphi) \in \mathbb{R} \times S^{1}$, where we have used that $u_{*}$ and $w+\alpha_{*} u_{*}$ are continuously differentiable.

The aim is to show that $w+\alpha_{*} u_{*} \equiv 0$ by showing that it vanishes on an open set. Then by unique continuation, it will be 0 everywhere. 
By (A3), there exists a $z_{0} \in \mathbb{R}$ such that $\int_{0}^{2 \pi} A_{0}\left(z_{0}, \varphi\right) d \varphi \neq 0$. By the intermediate value theorem, since $A_{0}$ is continuous and $A_{0}(z, \varphi) \rightarrow 0$ as $|z| \rightarrow \infty$, we may assume that $\int_{0}^{2 \pi} A_{0}\left(z_{0}, \varphi\right) d \varphi \neq$ $k \pi$ for all $k \in \mathbb{Z}$. By continuity of $\int_{0}^{2 \pi} A_{0}(\cdot, \varphi) d \varphi$, there exists an open interval $I \ni z_{0}$ such that $\int_{0}^{2 \pi} A_{0}(z, \varphi) d \varphi \neq k \pi$ for all $z \in I$.

We claim that for every $z \in I,\left(w+\alpha_{*} u_{*}\right)(z, \cdot)$ or $u_{*}(z, \cdot)$ vanishes on an interval in the $\varphi$ variable. To prove this, we argue by contradiction, and assume that there exists a $z_{1} \in I$ such that $\left(w+\alpha_{*} u_{*}\right)\left(z_{1}, \cdot\right)$ and $u_{*}\left(z_{1}, \cdot\right)$ do not vanish on any interval. Let $\varphi_{0} \in S^{1}$ be such that $\left(w+\alpha_{*} u_{*}\right)\left(z_{1}, \varphi_{0}\right) \neq 0$. We multiply (24) by the integrating factor

$$
\frac{\exp \left(2 i \int_{\varphi_{0}}^{\varphi} A_{0}\left(z_{1}, \psi\right) d \psi\right)}{\overline{w+\alpha_{*} u_{*}\left(z_{1}, \varphi\right)}},
$$

and note that the exponential in the numerator is always nonzero, while the denominator is nonzero in a neighbourhood of $\varphi_{0}$. Equation (24) for $z=z_{1}$ can then be rewritten as

$$
\frac{\partial}{\partial \varphi}\left(\frac{\exp \left(2 i \int_{\varphi_{0}}^{\varphi} A_{0}\left(z_{1}, \psi\right) d \psi\right) u_{*}\left(z_{1}, \varphi\right)}{\overline{\left(w+\alpha_{*} u_{*}\right)\left(z_{1}, \varphi\right)}}\right)=0,
$$

in a neighbourhood of $\varphi_{0}$, and hence

$$
\frac{\exp \left(2 i \int_{\varphi_{0}}^{\varphi} A_{0}\left(z_{1}, \psi\right) d \psi\right) u_{*}\left(z_{1}, \varphi\right)}{\overline{\left(w+\alpha_{*} u_{*}\right)\left(z_{1}, \varphi\right)}}=\frac{u_{*}\left(z_{1}, \varphi_{0}\right)}{\overline{\left(w+\alpha_{*} u_{*}\right)\left(z_{1}, \varphi_{0}\right)}}
$$

for $\varphi$ in this neighbourhood.

If $\varphi_{1}$ is another point such that $\left(w+\alpha_{*} u_{*}\right)\left(z_{1}, \varphi_{1}\right) \neq 0$, then the same argument can be used around $\varphi_{1}$ instead of $\varphi_{0}$. We now need to prove that (25) is valid with the same $\varphi_{0}$ for all $\varphi \in S^{1}$. For this, we need to extend (25) across the zeros of $\left(w+\alpha_{*} u_{*}\right)\left(z_{1}, \cdot\right)$. Let us first consider a point $\varphi_{2} \in S^{1}$ for which $\left(w+\alpha_{*} u_{*}\right)\left(z_{1}, \varphi_{2}\right)=0$ and $\partial_{\varphi}\left(w+\alpha_{*} u_{*}\right)\left(z_{1}, \varphi_{2}\right) \neq 0$. On each side of this zero, an equation (25) is valid, possibly with two different $\varphi_{0}$. Then

$$
u_{*}(z, \varphi)= \begin{cases}C_{1}(z) \exp \left(2 i \int_{0}^{\varphi} A_{0}\left(z_{1}, \psi\right) d \psi\right) \overline{\left(w+\alpha_{*} u_{*}\right)(z, \varphi)}, & \text { for } \varphi<\varphi_{2} \\ C_{2}(z) \exp \left(2 i \int_{0}^{\varphi} A_{0}\left(z_{1}, \psi\right) d \psi\right) \overline{\left(w+\alpha_{*} u_{*}\right)(z, \varphi)}, & \text { for } \varphi>\varphi_{2}\end{cases}
$$

in a neighbourhood of $\varphi_{2}$ and for $z=z_{1}$. Differentiating (26) with respect to $\varphi$ and taking limits from the left and right, we conclude that $C_{1}\left(z_{1}\right)=C_{2}\left(z_{2}\right)$ since $u_{*}$ and $w+\alpha_{*} u_{*}$ are $C^{1}$ functions. This shows that the formula (25) can be extended across zeros of $\left(w+\alpha_{*} u_{*}\right)\left(z_{1}, \cdot\right)$ for which $\partial_{\varphi}\left(w+\alpha_{*} u_{*}\right)\left(z_{1}, \cdot\right) \neq 0$.

Next, consider a point $\varphi_{3}$ for which $\left(w+\alpha_{*} u_{*}\right)\left(z_{1}, \varphi_{3}\right)=\partial_{\varphi}\left(w+\alpha_{*} u_{*}\right)(z, \varphi)=0$. Recall that we have assumed that $w+\alpha_{*} u_{*}$ is not entirely 0 on an interval containing $\varphi_{3}$. Then there are two mutually exclusive possibilities: Either

i) For every $\epsilon>0$, there exists a $z \in\left(z_{1}-\epsilon, z_{1}+\epsilon\right)$ and $a \delta>0$ such that for every $\varphi \in\left(\varphi_{3}-\delta, \varphi_{3}+\delta\right)$ it holds that $\left(w+\alpha_{*} u_{*}\right)(z, \varphi) \neq 0$ or $\nabla\left(w+\alpha_{*} u_{*}\right)(z, \varphi) \neq 0$, or

ii) There exists an $\epsilon>0$ such that for every $z \in\left(z_{1}-\epsilon, z_{1}+\epsilon\right)$ and every $\delta>0$ there exists $a \varphi \in\left(\varphi_{3}-\delta, \varphi_{3}+\delta\right)$ such that $\left(w+\alpha_{*} u_{*}\right)(z, \varphi)=0$ and $\nabla\left(w+\alpha_{*} u_{*}\right)(z, \varphi)=0$.

In case i), (26) is valid in a neighbourhood of $\varphi_{3}$, and a similar formula also holds for other $z$ close to $z_{1}$, and the formula can be extended across any zero of $\left(w+\alpha_{*} u_{*}\right)(z, \cdot)$ for which $\partial_{\varphi}\left(w+\alpha_{*} u_{*}\right)(z, \cdot) \neq 0$ for $z$ close to $z_{1}$. Since $u_{*}$ and $w+\alpha_{*} u_{*}$ are $C^{1}$ functions, $C_{1}$ and $C_{2}$ are also $C^{1}$ functions. An argument using the triangle inequality shows that the functions $C_{1}\left(z_{1}\right)=C_{2}\left(z_{1}\right)$.

In case ii), a continuity argument shows that $\left(w+\alpha_{*} u_{*}\right)\left(z, \varphi_{3}\right)=0$ and $\partial_{\varphi}\left(w+\alpha_{*} u_{*}\right)\left(z, \varphi_{3}\right)=$ 0 for each $z$ in an interval containing $z_{1}$. Notice that $w+\alpha_{*} u_{*}$ solves the second order elliptic linear PDE $-\partial_{z}^{2} u-\left(\partial_{\varphi}+i A_{0}(z, \varphi)\right)^{2} u=\lambda_{0} u$ in a domain containing this line segment, and that it satisfies Cauchy data on the segment. Uniqueness of the Cauchy problem [4] shows that $w+\alpha_{*} u_{*} \equiv 0$ in an open set, and in particular $\left(w+\alpha_{*} u_{*}\right)\left(z_{1}, \cdot\right)$ vanishes on a line segment, which is a contradiction. 
This shows that only case i) is possible, and the formula (25) can be extended across isolated points of the zero set of $\left(w+\alpha_{*} u_{*}\right)\left(z_{1}, \cdot\right)$, and

$$
u_{*}\left(z_{1}, \varphi\right)=C\left(z_{1}\right) \overline{\left(w+\alpha_{*} u_{*}\right)\left(z_{1}, \varphi\right)} \exp \left(2 i \int_{0}^{\varphi} A_{0}\left(z_{1}, \psi\right) d \psi\right)
$$

Note that $u_{*}\left(z_{1}, \cdot\right)$ and $\left(w+\alpha_{*}\right)\left(z_{1}, \cdot\right)$ are both $2 \pi$-periodic, and so

$$
C\left(z_{1}\right) \overline{\left(w+\alpha_{*} u_{*}\right)\left(z_{1}, \varphi_{0}\right)}\left(\exp \left(2 i \int_{0}^{2 \pi} A_{0}\left(z_{1}, \psi\right) d \psi\right)-1\right)=0
$$

for some $\varphi_{0}$ such that $\left(w+\alpha_{*} u_{*}\right)\left(z_{1}, \varphi_{0}\right) \neq 0$. Since $z_{1}$ was chosen such that $\int_{0}^{2 \pi} A_{0}\left(z_{1}, \psi\right) d \psi \neq k \pi$ for all $k \in \mathbb{Z}$, the only possibility is that $C\left(z_{1}\right)=0$. But then $u_{*}$ vanishes on an interval, contrary our assumption. This proves that our assumption that $\left(w+\alpha_{*} u_{*}\right)\left(z_{1}, \cdot\right)$ or $u_{*}\left(z_{1}, \cdot\right)$ does not vanish on an interval was wrong, an hence it must be true that $\left(w+\alpha_{*} u_{*}\right)(z, \cdot)$ or $u_{*}(z, \cdot)$ vanish on an interval for every $z \in I$. This is sufficient for proving that $u_{*}$ or $w+\alpha_{*} u_{*}$ vanish on a nonempty open set, by the following application of the Baire category theorem:

Proposition 1. Let $I$ be an interval in $\mathbb{R}$. If $f: I \times I \rightarrow \mathbb{C}$ is continuous and for every $x \in I$ there exists an interval $I_{x} \subset I$ such that $f(x, y)=0$ for every $y \in I_{x}$, then $f \equiv 0$ on a nonempty open subset of $I \times I$.

Proof. For $p, q \in \mathbb{Q} \cap I$, let $B(p, q)=\left\{x ;[p, q] \subset I_{x}\right\}$, and note that by assumption, $\cup_{p, q} B(p, q)=I$. Since $f$ is continuous, the sets $B(p, q)$ are closed. By the Baire category theorem, at least one of the sets $B(p, q)$ contains an interval $\left[x_{1}, x_{2}\right]$, since otherwise the interval $I$ would be a countable union of nowhere dense sets. This proves that $f(x, y)=0$ for $(x, y) \in\left(x_{1}, x_{2}\right) \times(p, q)$, which is a nonempty open subset of $I \times I$.

By applying Proposition 1 to $w+\alpha_{*} u_{*}$ or $u_{*}$ we find that $w+\alpha_{*} u_{*}$ or $u_{*}$ has to vanish on an open set, and by unique continuation, it must vanish entirely on $\mathbb{R} \times S^{1}$. But $u_{*}$ is the eigenfunction, so it cannot vanish everywhere, so the only possibility is that $w+\alpha_{*} u_{*}$ vanishes on $\mathbb{R} \times S^{1}$. Hence $\alpha_{k}=0$ for $k=1, \ldots, 4 m-2$, and so $F_{k}^{\prime}\left(A_{0}\right), k=1, \ldots, 4 m-2$ are linearly independent. The proof can now be concluded as follows:

Define $\mathcal{F}: X_{\beta} \rightarrow \mathbb{C}^{4 m-2}$ by

$$
\mathcal{F}(A):=\left(F_{1}(A), \ldots, F_{4 m-2}(A)\right)^{T} .
$$

By the linear independence of the components of $\mathcal{F}^{\prime}\left(A_{0}\right)$, we have a decomposition

$$
X_{\beta}=\left(\operatorname{ker} \mathcal{F}^{\prime}\left(A_{0}\right)\right) \oplus \mathcal{M}
$$

where $\mathcal{M}$ is $(4 m-2)$-dimensional. Moreover, the map $\mathcal{F}^{\prime}\left(A_{0}\right)$ is surjective. For $A \in X_{\beta}$ we write $A=A_{1}+A_{2}$, where $A_{1} \in \operatorname{ker} \mathcal{F}^{\prime}(0)$ and $A_{2} \in \mathcal{M}$.

Finally, we define $\mathcal{G}:\left(\operatorname{ker} \mathcal{F}^{\prime}\left(A_{0}\right)\right) \times \mathcal{M} \rightarrow \mathbb{C}^{4 m-2}$ by $\mathcal{G}\left(A_{1}, A_{2}\right)=\mathcal{F}\left(A_{1}+A_{2}\right)$. The Fréchet derivative at $(0,0)$ with respect to $A_{2}$, which we denote by $\mathcal{G}_{2}^{\prime}(0,0)$ is invertible, and so by the implicit function theorem, the equation $\mathcal{G}\left(A_{1}, A_{2}\right)$ is solvable for $A_{2}$ in terms of $A_{1}$, and this equation defines a smooth manifold of codimension $4 m-2$ in a neighbourhood of $A=A_{0}$.

\section{ACKNOWLEDGEMENTS}

The second author would like to thank Ira Herbst for introducing her to the area of embedded eigenvalues, and to Amol Sasane, Gianne Derks and Mikael Persson Sundqvist for useful discussions. A helpful discussion on mathoverflow with Fedor Petrov is also gratefully acknowledged by the second author. Finally, both authors would like to thank the anonymous reviewer for the useful comments. Sara Maad Sasane's research was supported by Vinnova (grant no. 2011-03440). 


\section{REFERENCES}

[1] Agmon, S., Herbst, I., And Maad Sasane, S. Persistence of embedded eigenvalues. Journal of Functional Analysis 261 (2011), 451-477.

[2] Agmon, S., Herbst, I., And Skibsted, E. Perturbations of embedded eigenvalues in the generalized $N$-body problem. Comm. Math. Phys. 122 (1989), 411-438.

[3] Akhiezer, N., and Glazman, I. Theory of linear operators in Hilbert. Frederick Ungar publishing co, 1961.

[4] Aronszajn, N. A unique continuation theorem for solutions of elliptic partial differential equations or inequalities of second order. J. Math. Pures Appl. 36 (1957), 235-249.

[5] Astaburuaga, M., Briet, P., Bruneau, V., Fernández, C., and Raikov, G. Dynamical resonances and SSF singularities for a magnetic Schrödinger operator. Serdica Mathematical Journal 34 (2008), 179-218.

[6] Bögli, S., Brown, B., Marletta, M., Tretter, C., and Wagenhofer, M. Guranteed resonance enclosures and exclosures for atoms and molecules. Proc. R. Soc. A..

[7] Bruneau, V., Khochman, A., and Raikov, G. Perturbation of a magnetic schrödinger operator near an embedded infinite-multiplicity eigenvalue. In Spectral and scattering theory for quantum magnetic systems, P. Briet, F. Germinet, and G. Raikov, Eds., Contemporary mathematics. American mathematial society, 2009, pp. $47-61$.

[8] Colin de Verdière, Y. Pseudo Laplacians I. Ann. Inst. Fourier 32 (1982), 275-286.

[9] Colin de Verdière, Y. Pseudo Laplacians II. Ann. Inst. Fourier 33 (1983), 87-113.

[10] Cruz-Sampedro, J., Herbst, I., and Martínez-Avendaño, R. Perturbations of the Wigner-von Neumann potential leaving the embedded eigenvalue fixed. Ann. Henri Poincaré 3 (2002), 331-345.

[11] Derks, G., MaAd, S., And Sandstede, B. Perturbations of embedded eigenvalues for the bilaplacian on a cylinder. Discrete Contin. Dyn. Syst. (2008).

[12] Derks, G., Maad Sasane, S., and Sandstede, B. Perturbations of embedded eigenvalues for the planar bilaplacian. F. Funct. Anal. 260, 2 (2011), 340-398.

[13] Elton, D. The local structure of zero mode producing magnetic potentials. Commun. Math. Phys. 229 (2002), $121-139$.

[14] Gilbarg, D., and Trudinger, N. S. Elliptic Partial Differential Equations of Second Order. Springer-Verlag, 1998.

[15] Henry, D. Geometric theory of semilinear parabolic equations. Springer Verlag, 1981.

[16] Kato, T. Perturbation theory for linear operators, vol. 132 of Grundlehren der Mathematischen Wissenschaften. Springer-Verlag, Berlin Heidelberg New York, 1966.

[17] Mielke, A., and Zelik, S. V. Infinite-dimensional trajectory attractors of elliptic boundary-value problems in cylindrical domains. Russian Math. Surveys 57 (2002), 753-784.

[18] Nazarov, S. Enforced stability of an eigenvalue in the continuous spectrum of a waveguide with an obstacle. Comput. Math. Math. Phys. 52, 3 (2012), 448-464.

[19] PAZY, A. Semigroups of linear operators and applications to partial differential equations, vol. 44 of Applied mathematical sciences. Springer-Verlag, New York, 1983.

[20] Peterhof, D., Sandstede, B., and Scheel, A. Exponential dichotomies for solitary-wave solutions of semilinear elliptic equations on infinite cylinders. Journal of Differential Equations 140 (1997), 266-308.

[21] Phillips, R., And Sarnak, P. On cusp forms for co-finite subgroups of PSL(2, $\mathbb{R})$. Invent. Math. 80 (1985), 339-364.

[22] Phillips, R., And Sarnak, P. Automorphic spectrum and Fermi's golden rule. Festschrift on the occasion of the 70th birthday of Shmuel Agmon., J. Anal. Math. 59 (1992), 179-187.

[23] Phillips, R., and Sarnak, P. Perturbation of the Laplacian on automorphic functions. J. Amer. Math. Soc. 5 (1992), 1-32.

[24] Reed, M., And Simon, B. Methods of modern mathematical physics, I-IV. Academic Press, 1972-1978.

[25] Simon, B. Resonances in $N$-body quantum systems with dilation analytic potentials and the foundation of time-dependent perturbation theory. Ann. of Math. $9^{77}$ (1973), 247-274.

Ari Laptev, Imperial College London and St. Petersburg State University

E-mail address: a.laptev@imperial.ac.uk

Sara Maad Sasane, Lund University

E-mail address: sara@maths.lth.se 